OPEN ACCESS

Edited by:

Dipesh Dhakal,

Sun Moon University, South Korea

Reviewed by:

Juan Carlos Aon,

GlaxoSmithKline, United States

Anil Shrestha,

Ewha Womans University,

South Korea

${ }^{*}$ Correspondence:

Huawei Zhang

hwzhang@zjut.edu.cn

Hong Wang

hongw@zjut.edu.cn

Specialty section:

This article was submitted to

Microbial Physiology and Metabolism,

a section of the journal

Frontiers in Microbiology

Received: 11 August 2018

Accepted: 04 February 2019

Published: 26 February 2019

Citation:

Pan R, Bai X, Chen J, Zhang H and Wang $H$ (2019) Exploring Structural Diversity of Microbe Secondary Metabolites Using OSMAC Strategy: A Literature Review. Front. Microbiol. 10:294. doi: 10.3389/fmicb.2019.00294

\section{Exploring Structural Diversity of Microbe Secondary Metabolites Using OSMAC Strategy: A Literature Review}

\author{
Rui Pan ${ }^{1}$, Xuelian Bai ${ }^{2}$, Jianwei Chen ${ }^{1}$, Huawei Zhang ${ }^{1 *}$ and Hong Wang ${ }^{1 *}$ \\ ${ }^{1}$ School of Pharmaceutical Sciences, Zhejiang University of Technology, Hangzhou, China, ${ }^{2}$ College of Life \\ and Environmental Sciences, Hangzhou Normal University, Hangzhou, China
}

Microbial secondary metabolites (MSMs) have played and continue to play a highly significant role in the drug discovery and development process. Genetically, MSM chemical structures are biologically synthesized by microbial gene clusters. Recently, however, the speed of new bioactive MSM discovery has been slowing down due to consistent employment of conventional cultivation and isolation procedure. In order to alleviate this challenge, a number of new approaches have been developed. The strategy of one strain many compounds (OSMAC) has been shown as a simple and powerful tool that can activate many silent biogenetic gene clusters in microorganisms to make more natural products. This review highlights important and successful examples using OSMAC approaches, which covers changing medium composition and cultivation status, co-cultivation with other strain(s), adding enzyme inhibitor(s) and MSM biosynthetic precursor(s). Available evidences had shown that variation of cultivation condition is the most effective way to produce more MSMs and facilitate the discovery of new therapeutic agents.

Keywords: OSMAC strategy, microbe secondary metabolite, structural diversity, medium composition, co-cultivation, epigenetic modification

\section{INTRODUCTION}

Microbial secondary metabolites (MSMs) have been recognized as the primary source of new compounds for drug discovery and development (Gunatilaka, 2006; Rateb et al., 2011b; Deng et al., 2013). Traditional chemical investigation of microorganism mainly focuses on extraction and isolation of structurally and highly active compounds from fermentation broth and mycelium. However, these processes are becoming inefficient due to high rate of the re-discovery of known MSMs. It is commonly believed that a large portion of microbial gene clusters are silenced under standard fermentation conditions (Scherlach and Hertweck, 2009; Wasil et al., 2013). By mining microbial genome and targeting biosynthetic gene clusters of MSM, researchers can exploit the potential of microbes in a more objective way, such as knocking down, introduction or heterologous expression of microbial genes, regulation of promoters, induction of mutations, or changing cultivation conditions to stimulate MSM genes expression (Schneider et al., 2008). Variation of 
cultivation condition has been deemed to be the simplest and most effective strategy, which is termed as "one strain many compounds (OSMAC)" by professor Zeeck and coworkers (Bode et al., 2002). On basis of extensive literature search, important and successful examples using OSMAC strategy are summarized in this review, which consists of variation of medium, changing cultivation condition, cocultivation with other strain(s), adding epigenetic modifier(s) or biosynthetic precursor(s).

\section{VARIATION OF MEDIUM}

Culture medium has a greater effect not only on microbe growth but also on metabolism. It has been reported that $\mathrm{C} / \mathrm{N}$ ratio, salinity, and metal ion can regulate the degree and pattern of MSM gene expression and result in production of various secondary metabolites.

\section{Medium Composition}

Generally, carbon and nitrogen sources are major components in the culture medium. The carbon source not only provides the basis for building biomass and represents the source of energy for all heterotrophs but also delivers carbon units for secondary metabolites. The nitrogen source is required for the synthesis of essential proteins and nucleic acids, and likewise $N$-containing units for secondary metabolites. The type of used carbon and nitrogen sources is known to have a significant influence on microbial secondary metabolism (Ruiz et al., 2009; Singh et al., 2017). Furthermore, the $\mathrm{C} / \mathrm{N}$ ratio is one of important factors that affect fermentation products (Karakoç and Aksöz, 2004; Brzonkalik et al., 2012; Dinarvand et al., 2013). Notably, the consumption of carbon and nitrogen-based medium components can greatly affects the $\mathrm{pH}$ of the cultivation broth, e.g., by formation of organic acids or the accumulation of basic ammonium. Thus, microorganisms cultured in medium containing different components may exhibit differently adapted metabolism and express specific sets of biosynthetic genes, which produced a differential biosynthesis of specialized metabolites (Ma et al., 2009).

One marine-derived strain Asteromyces cruciatus 763 was shown to produce a new pentapeptide lajollamide A (1), when cultivated in the Czapek-Dox broth contained arginine solely as nitrogen source rather than $\mathrm{NaNO}_{3}$, which was missed in the normal Czapek-Dox medium (Gulder et al., 2012). One sediment-derived Aspergillus niger BRF-074 produced a novel furan ester derivative (2), a compound has toxicity acidity against HCT-116 cancer cell line (Uchoa et al., 2017), when cultivated in MPDB (malt peptone dextrose broth) medium. But this compound failed to appear in PDB (potato dextrose broth) or PDYB (potato dextrose yeast broth) media. A fungus Aspergillus sp. from Waikiki Beach (Honolulu, HI), generated six isotopically labeled metabolites (3-8) when grown on the deuterium-enriched Czapek broth (Wang et al., 2015a), whereas this strain was found to metabolite a novel prenylated indole alkaloid, waikialoid A (9) when cultivated in PDB medium. Bioassay results indicated that compound $\mathbf{9}$ possessed potent inhibitory effect on biofilm formation of Candida albicans with an $\mathrm{IC}_{50}$ value of $1.4 \mu \mathrm{M}$ (Wang Q.X. et al., 2012).
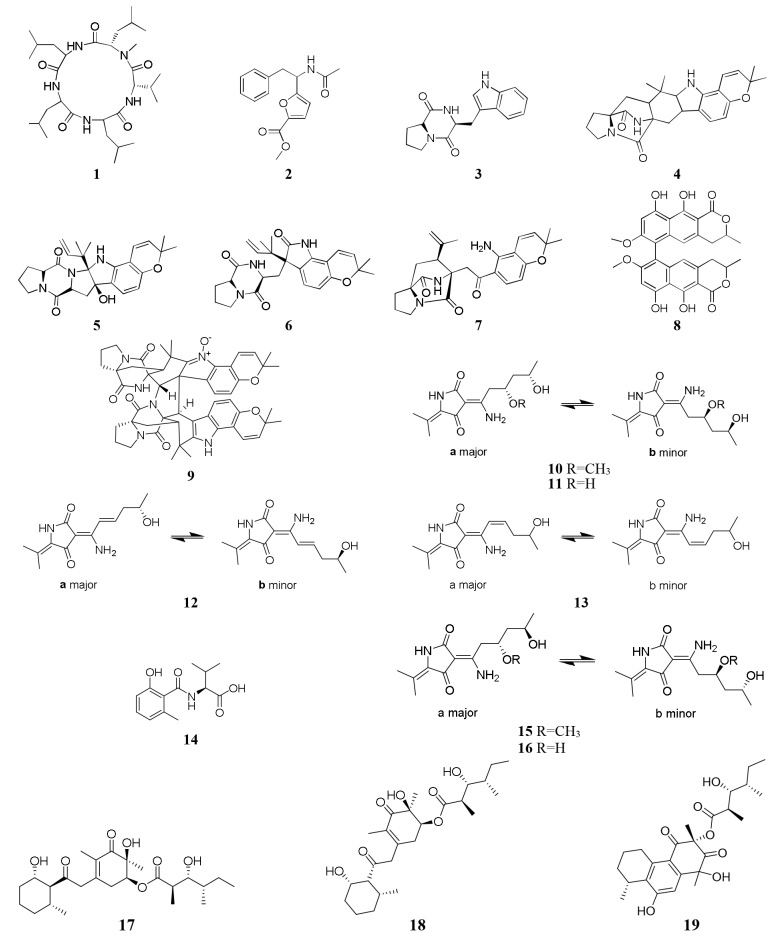

Five new polyketides (10-14) were detected in the crude extract of rice-based medium of a marine-derived Cladosporium sphaerospermum 2005-01-E3 (Wu et al., 2014). Another two new hybrid polyketides (15-16) were accessed when the same strain was fermented on the soybean flour (Yu et al., 2015). The organic extract of Dothideomycete sp. CRI7 was elaborated by four comparative medium. The strain growing in PDB made with potato tubers led to the isolation of azaphilone derivatives (17-18) and a novel tricyclic polyketide (19). Only compound 19 exhibited a broad spectrum of cytotoxic activities (Senadeera et al., 2012). It is interesting that MSM production by strain CR17 was sensitive to sources of potato and malt extract used for the preparation of PDB and Czapek malt media, respectively. Three new polyketides (20-22) were produced when strain CR17 was grown in PDB broth prepared from a commercial potato powder instead of fresh tubers of potato, while this strain produced several other compounds (20-21 and 23-25) in Czapek malt medium. Compound 24 exhibited cytotoxic activity against cancer cell lines MOLT-3, HuCCA-1and A549 with IC $_{50}$ values of $17.4,48.1,46.5 \mu \mathrm{g} / \mathrm{mL}$, respectively (Hewage et al., 2014). One fungus strain of Fusarium tricinctum isolated in Beni-Mellal, which can colonize the rhizomes of Aristolochia paucinervis, could afforded three new fusarielins (26-28). But these metabolites were not detected when cultivated in normal rice medium supplemented with fruit and vegetable juice. Bioassay results suggested that compound $\mathbf{2 6}$ possessed cytotoxic effect on human ovarian cancer cell line A2780 with an $\mathrm{IC}_{50}$ value of $12.5 \mu \mathrm{M}$ (Hemphill et al., 2017). A new diketopiperazine (29) was isolated from Eurotium rubrum MPUC136 cultured by 
wheat medium, which displayed more powerful bioactivity than the Czapek-Dox agar medium, and shown to have cytotoxicity against $\mathrm{B}_{16}$ melanoma cell line with an $\mathrm{IC}_{50}$ value of $60 \mu \mathrm{M}$ (Kamauchi et al., 2016).
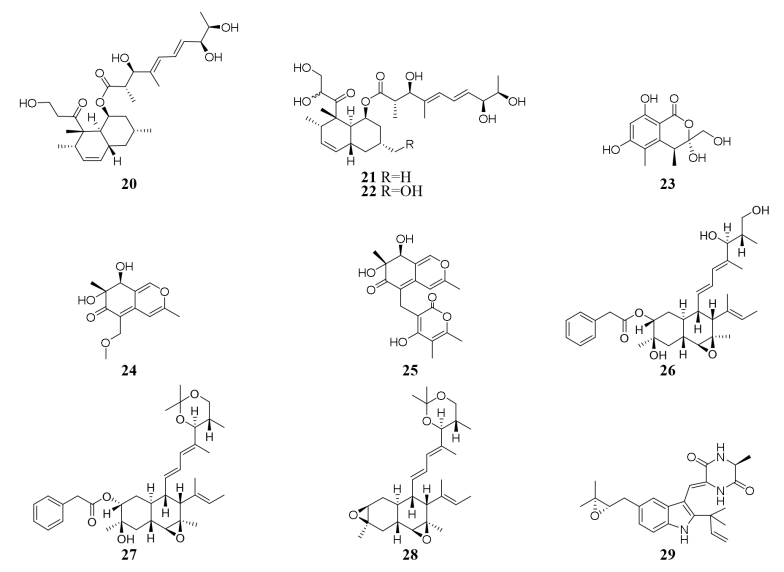

HPLC analysis of crude extracts of an actinomycete strain Lentzea violacea AS08 indicated different composition in three media including CYPS (casein yeast peptone), SCP-1 (starch casein peptone), and SC (starch casein) (Hussain et al., 2017). Only one new eudesmane sesquiterpenoid (30) and a new analog of virginiae butanolide E (31) were detected in SC medium, and compound 30 exhibited moderate cytotoxic effect on HCT-116 and A549 tumor cell lines with IC $_{50}$ values of 19.2 and 22.3 $\mu \mathrm{M}$, respectively. One rhizosphere fungus Paraphaeosphaeria quadriseptata produced a known C18 polyketide monocillin I together with several analogs when incubated in PDA medium constituted with tap water (Wijeratne et al., 2004). However, the same fungal strain could make six new trihydroxybenzene lactones, cytosporones F-I (32-37), when the tap water was changed as distilled water (Paranagama et al., 2007). Similarly, one new naphthalopyran compound (38), which possesses an unusual oxygenated aromatic structure with a lactone bridge, could be metabolized by the fungus $P$. hordei grown on plant tissue agar such as macerated tulip and yellow onion, oatmeal and red onion, while it was not detected in CYA (caffeic acid agar), MEA (malt extract agar), and YES (yeast extract with supplements) media (Overy et al., 2005). When cultivated in rice medium, a hard coral-derived fungus Scopulariopsis sp. from the coastline of Red Sea was shown to afford six secondary metabolites including xanthone derivatives (3940), phenolic bisabolane-type sesquiterpenes (41-42), one new alkaloid (43) and one new $\alpha$-pyrone derivative (44) (Elnaggar et al., 2016). Interestingly, this strain could biosynthesize a new naphthoquinone derivative (45) and two new triterpenoids (4647) in the protein-rich white bean medium (Elnaggar et al., 2017).

Chemical investigation of one marine-derived strain Streptomyces sp. C34 grown on ISP2 (yeast malt extract agar) medium led to the isolation of four new ansamycin-type polyketides (48-49). But only compounds 48, 50, and 51 could be extracted from modified ISP2 medium, which contained glycerol rather than glucose. Bioassay results indicated that
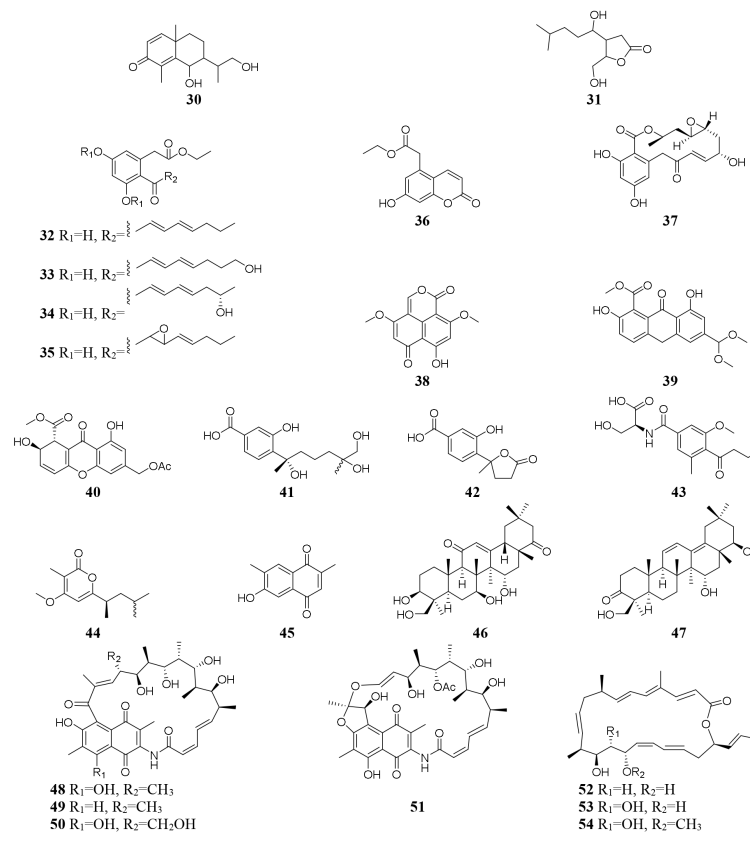

$33 \mathrm{R}_{1}=\mathrm{H}, \mathrm{R}_{2}=$ 年

$34 \mathrm{R}_{1}=\mathrm{H}, \mathrm{R}_{2}=$

$35 \mathrm{R}_{1}=\mathrm{H}, \mathrm{R}_{2}=\xi \mathrm{q}^{2}$
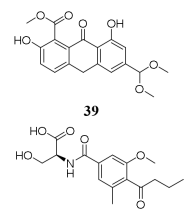

43
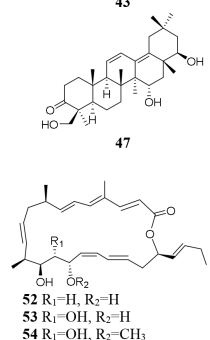

compound $\mathbf{5 1}$ had a selective inhibitory effect on S. aureus ATCC 25923 with a MIC value of $0.05 \mu \mathrm{g} / \mathrm{mL}$ (Rateb et al., 2011a). The utilization of a defined medium to cultivate strain C34 resulted in the observation of three novel 22-membered lactone polyketides (52-54) (Reid et al., 1995). Compounds 50-52 possessed strong antibacterial activities against L. monocytogenes and B. subtilis with MIC values range from 3 to $6 \mu \mathrm{g} / \mathrm{mL}$ and against $S$. aureus with MIC values of $<1 \mu \mathrm{g} / \mathrm{mL}$ (Rateb et al., 2011a). Four media applied to strain Streptomyces sp. CS resulted in production of various natural products including three new macrolides (55-57) from YMG agar medium, five new 16-membered macrolides (58-62) from ISP2 broth, five novel polyketides (63-67) from sterilized Waksman Synthetic medium and three new naphthomycins (68-70) from oatmeal medium. Compounds 55 was shown to have inhibitory effect on Fusarium moniliforme with a MIC value of $300 \mu \mathrm{g} / \mathrm{mL}$ and compounds 58-62 exhibited cytotoxicity toward the MDA-MB435 human cancer cell line with $\mathrm{IC}_{50}$ values of $4.2,4.5,5.5,3.8$, and 11.4 mM, respectively (Lu and Shen, 2003, 2004; Li et al., 2008, 2010; Yang et al., 2012). Streptomyces sp. ML55 in a medium consisting of glycerin, molasses, casein, polypeptone led to the isolation of three novel antimycins, JBIR-02 (71), JBIR-06 (72), and JBIR-52 (73), while this strain had capacity to produce two novel depsipeptides (74-75) in GYM medium (Ueda et al., 2007, 2008; Kozone et al., 2009; Li X. et al., 2013). An ant-derived actinomycete Streptomyces sp. 1H-GS5 was found to produce one new spectinabilin derivative (76) when cultivated in the medium consisting of corn starch $10 \%$, soybean powder $1 \%$, cotton flour $1 \%$, $\alpha$-amylase $0.02 \%, \mathrm{NaCl} 0.1 \%, \mathrm{~K}_{2} \mathrm{HPO}_{4} 0.2 \%, \mathrm{MgSO} 4 \cdot 7 \mathrm{H}_{2} \mathrm{O}$ $0.1 \%, \mathrm{CaCO}_{3} 0.7 \%$, cyclohexanecarboxylic acid $0.1 \%, \mathrm{pH} 7.0$, while this stain made another new cytotoxic spectinabilin (77) when reducing the proportion of nutrients (Liu S. et al., 2015; Liu C. X. et al., 2016). 


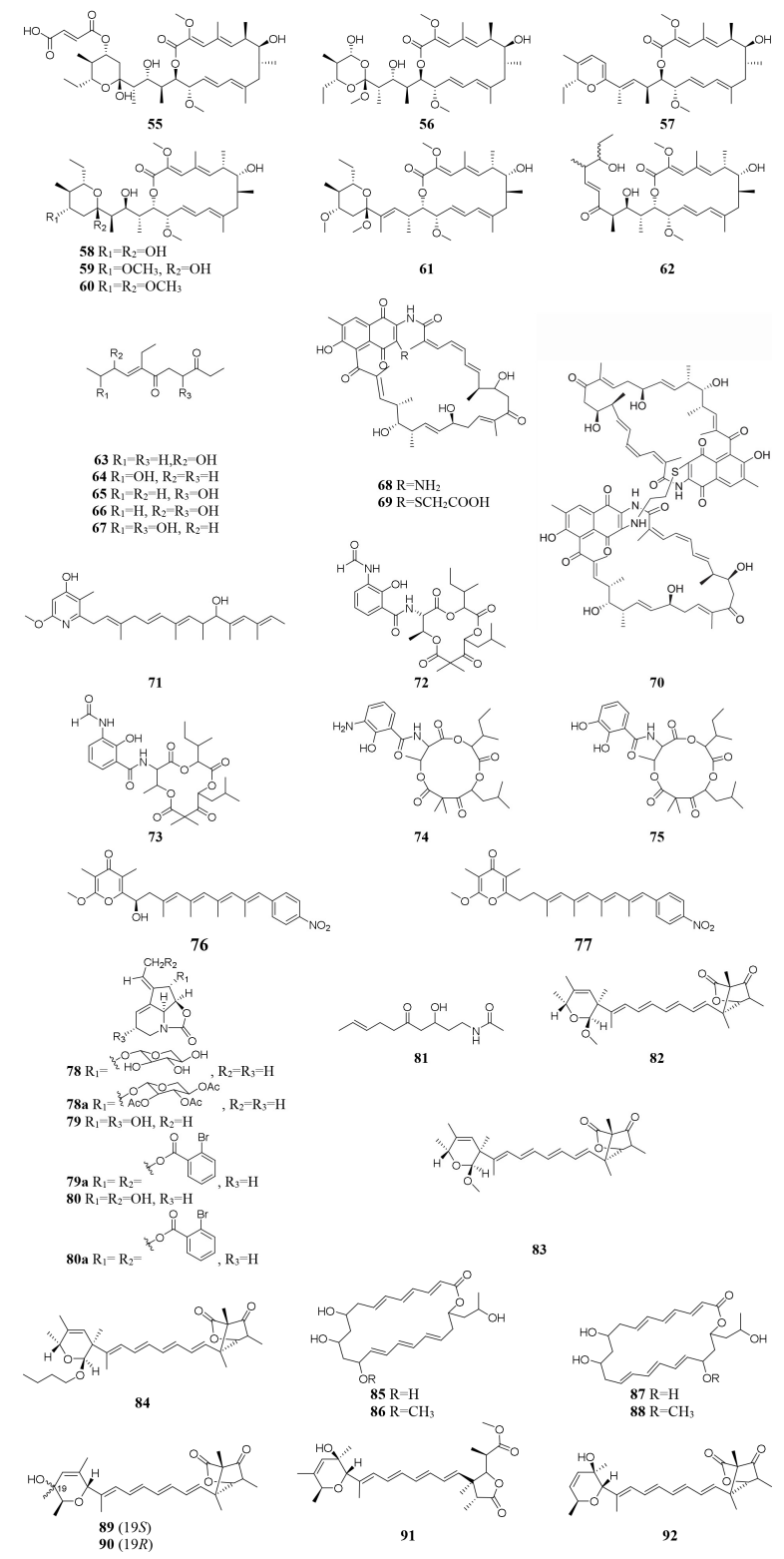

When cultured in an oat bran medium, one strain Streptomyces sp. A1 was found to produce rubromycin derivatives, while other three known compounds were biosynthesized in a mannitol/soybean meal medium and three new congeners (78-80) and streptenol E (81) in medium (degreased soybean meal $2 \%$, mannitol $2 \%$, agar $2 \%$ ) with soil as an addition, provide. Compound $\mathbf{8 1}$ had significant cytostatic effect on four tumor cell lines including HMO2, HEP G2, MCF7 and Kato III with GI $_{50}$ values (the concentration that causes $50 \%$ growth inhibition) of $0.15,0.3,10$, and $0.7 \mu \mathrm{M}$, respectively (Puder et al., 2001). Phytochemical study of one filamentous soil fungus, Talaromyces wortmannii, cultivated in maize culture medium, led to the separation of three new polyketones (82-84), which were absent in rice or dextrose agar media. Compounds 82-84 displayed inhibitory activities against NFRD (fumarate reductase) with $\mathrm{IC}_{50}$ values of 8.8, 11, and $13 \mu \mathrm{M}$, respectively (Liu W. C. et al., 2016).
Interestingly, this stain was found to produce four novel 22-membered macrolides (85-88) (Dong et al., 2006) and four novel tetraene lactones (89-92) (Dong et al., 2009) when grown in the still-cultured medium (2.5\% soybean meal and $97.5 \%$ rice). Compounds 85-88 exhibited in vitro moderate cytotoxic activities against human cancer cell lines (HCT-5, HCT115, A549, MDA-MB-231, and K562) with $\mathrm{IC}_{50}$ values range from 28.7 to $130.5 \mu \mathrm{M}$, while compounds 89-92 showed potent inhibitory effects on cathepsin B.

\section{Salinity}

Salinity is an important factor in determining many aspects of the chemistry of natural water and of biochemical process within cultivation system, and is a thermodynamic state variable that, along with temperature and pressure, governs physical characteristics like the osmotic pressure and enzymes involved in microbial growth and metabolism (Blunt et al., 2015). Suitable salinity is needed for normal microbial growth and high osmotic pressure makes cells dehydrated and affects microbial biochemical reactions (Poolman and Glaasker, 1998; Wang Y. et al., 2011).

Microorganisms exposed to different types of media supplemented with various halogens maybe trigger their synthesis pathway to restore osmotic imbalance, thus activating different hidden MSM biosynthetic gene clusters. Compare to that grown in seawater, one marine-derived fungus Aspergillus unguis CRI282-03 was shown to produce new brominated depsidones (93-95) and two new orcinol derivatives (96-97) in $\mathrm{KBr}$ medium and a new depsidone (98) in KI broth (Sureram et al., 2013). Bioassay results indicated that compounds $\mathbf{9 5}$ and 96 possesses aromatase inhibitory effects (Sureram et al., 2012). Nine new polyketides (99-107), which were absent in the broth contained KI or deionized water, were produced by the fungus Dothideomycete sp. CRI7 isolated from Tiliacora triandra when cultivated in the medium supplemented with $\mathrm{KBr}$ and seawater (Wijesekera et al., 2017).

Chemical investigation of one symbiotic stain Aspergillus sp. D from Edgeworthia chrysantha led to isolation of five known heterocyclic alkaloids from normal Czapek medium, while a new meroterpenoid (108) and four known analogs were obtained from Czapek medium with $3 \%$ salty (Zhang et al., 2018a,b). One mangrove-derived endophyte Wallemia sebi PXP-89 cultivated in $10 \% \mathrm{NaCl}$ broth produced a new cyclopentanol pyridine alkaloid (109), which was not detected in normal medium (Peng et al., 2011). When cultivated in medium containing $10 \%$ sea salt, strain Spicaria elegans KLA03 was shown to biosynthesize a new antimicrobial diacrylic acid (110) (Wang F. Z. et al., 2011). Strain Streptomyces sp. DSM 14386 could metabolize five new compounds (111-115) in $1.5 \% \mathrm{NaCl}$ medium, while this strain produced two brominated congeners (116-117) in 1.5\% NaBr medium. Antimicrobial tests showed that compounds $\mathbf{1 1 3}$ and $\mathbf{1 1 7}$ displayed potent antibiotics against MRSA (methicillin-resistant Staphylococcus aureus) with the same MIC values of $16 \mu \mathrm{g} / \mathrm{mL}$, and compound 117 also had strong activity toward Mycobacterium smegmatis $\left(\mathrm{IC}_{80}=2\right.$ $\mu \mathrm{g} / \mathrm{mL}$ ) (Onaka, 2017). Two rare epidithiodiketopiperazines, gliovirin and pretrichodermamide $\mathrm{A}$, were detected in $1.5 \% \mathrm{NaCl}$ 
broth of a marine-derived Trichoderma sp. TPU199, while this strain produced a new iodo derivative (118) from freshwater medium with $3.0 \% \mathrm{NaI}$ and $3.0 \% \mathrm{NaBr}$ as well as 5-bromo-5deoxy derivative (Yamazaki et al., 2015a).

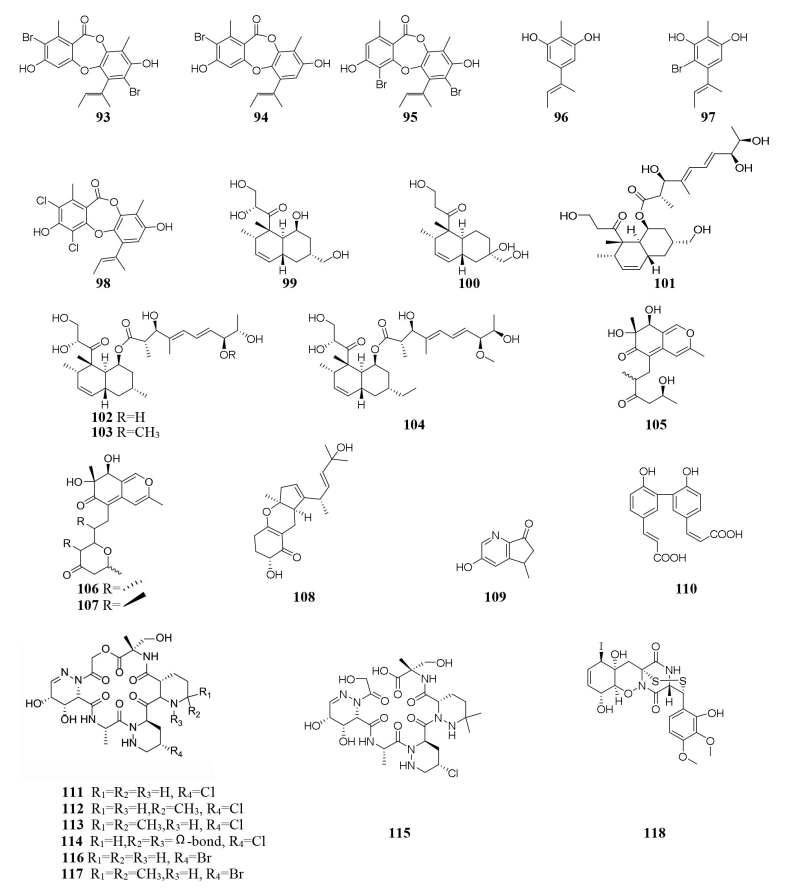

\section{Metal Ion}

Metal ion affects physiological structure and function of microorganism. The interaction between metal ion and microbe is usually assumed in three pathways, including causing reactions in cells, conserving energy in the process of dissimilation, and assimilating reactions (Thorneley, 1990).

One marine-derived strain Ascotricha sp. ZJ-M-5 was shown to produce a new 3,4-split ring lanolin alkyl triterpene (119) and a new cyclonerols derivative (120), when cultivated in eutrophic medium made up with sea salt (Xie et al., 2013a,b). However, three new caryophyllene derivatives (121-123) were detected in modified Czapek Dox medium, while compound 122 was absent in the fermentation broth without $\mathrm{Mg}^{2+}$ (Wang W.J. et al., 2014). Strain Aspergillus sclerotiorum C10WU derived from hydrothermal vent sediment in Taiwan (China) could produce three new alkaloids (124-126) under normal medium. However, this strain metabolized one unelucidated compound due to the low amount available together with aspochracin when grown in the stressed culture medium with $\mathrm{Cu}^{2+}$ as a supplement. Likewise, two compounds, namely deoxytryptoquivaline and tryptoquivaline A (127-128), were purified from the normal extract of $A$. clavatus C2WU, while only metabolite 129 was found in normal medium containing $\mathrm{Cu}^{2+}$ and $\mathrm{Cd}^{3+}$ (Jiang et al., 2014). A novel antibacterial cyclodepsipeptide, named NC-1 (130), was produced by a red soil-derived strain Streptomyces sp. FXJ1.172 when cultured in GYM (glucoseyeast extract-malt extract) medium added with ferric ion (Liu M. et al., 2016).
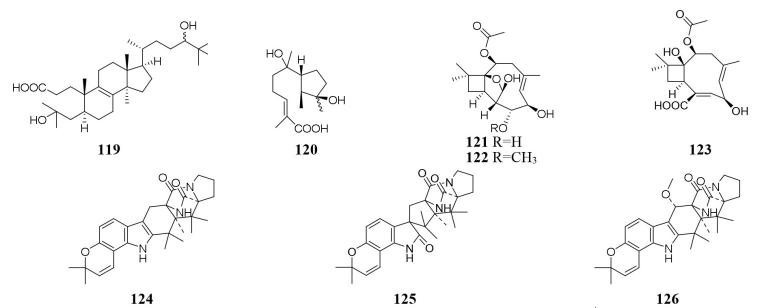

124

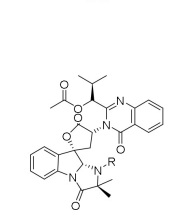

$127 \mathrm{R}=\mathrm{H}$
$128 \mathrm{R}=\mathrm{OH}$
126

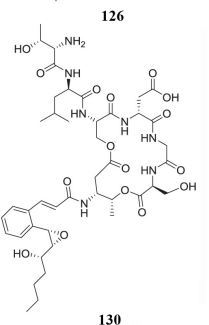

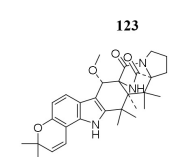

\section{CULTIVATION CONDITION}

Suitable cultivation conditions, such as appropriate temperature, $\mathrm{pH}$, oxygen concentration, and cultivation status, are essential for the growth and biochemical reactions of microorganisms. However, many biosynthetic genes of MSMs are not expressed under normal culture conditions, thus it is essential to change the cultivation condition to activate these silent gene clusters to diversify their MSMs.

\section{Temperature}

Chemical diversity of MSM is directly influenced by microbe enzyme activity, which is susceptible to cultivation temperature. The normal function of microbial enzyme is dependent on appropriate temperature. Generally, the higher the cultivation temperature is, the faster the enzyme deactivation rate will be (Feller et al., 1994). For example, when the temperature was lower than $30^{\circ} \mathrm{C}$, secondary metabolites of an uncoded strain Streptomyces sp. were composed of chlortetracycline, while only tetracycline was synthesized when cultivation temperature went up to $35^{\circ} \mathrm{C}$ (Cui et al., 1996).

\section{$\mathrm{pH}$}

During microbe fermentation process, the decomposition and utilization of nutrients as well as the accumulation of secondary metabolites usually causes the variation of medium $\mathrm{pH}$ (Gibson et al., 1988; Tan et al., 1998). It affects not only the activity of each enzyme, but also the surface charge of the membrane. The nature and permeability of cell membrane could change the rate of utilization of substrate, thus affecting the growth of microorganisms and biosynthesis of final products. Chemical study of one desert-derived strain Nocardiopsis alkaliphila nov. YIM-80379 led to isolation of two new pyran-2-one derivatives (131-132) when cultivated on Gause's synthetic agar slants with $\mathrm{pH}=10$. However, the neutral medium was unsuitable for its growth (Hozzein et al., 2004; Wang et al., 2013c). Acidic medium $(\mathrm{pH}=5)$ dramatically increased the production of bioactive compounds of a mangrovederived fungus Rhytidhysteron rufulum AS21B, including two 
new antitumor spirobisnaphthalenes (133-134). However, these compounds were not detected in neutral medium (Siridechakorn et al., 2017).

\section{Oxygen Concentration}

Changes in oxygen supply can affect the biochemical reactions and activate different set of functional gene clusters for different secondary metabolites production (Sato, 1990). For example, $\left[{ }^{13} \mathrm{C}\right]$-labeled acetates and a small amount of $\left[{ }^{18} \mathrm{O}_{2}\right]$ were used to investigate the biosynthetic pathway of aspinonene (135) in the culture broth of Aspergillus ochraceus DSM-7428. It is interesting that aspyrone (136) was produced by increasing dissolved oxygen concentration during fermentation, accompanied by reduced amounts of compound $\mathbf{1 3 5}$ under an oxygen enriched atmosphere (Fuchser et al., 1995).
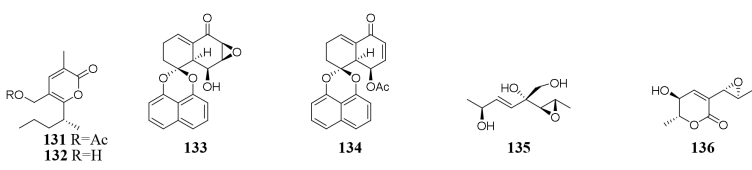

\section{Cultivation Status}

A growing body of evidence has indicated that cultivation status can directly affect microbe metabolic process, including solid or liquid, static or dynamic. Compared with solid and static cultivation, liquid and dynamic modes not only ensure the full contact of microorganisms and nutrients, but also affect their biochemical reactions by changing oxygen supply and activating functional gene clusters. Till now, MSMs from 12 genera had been investigated under different fermentation status, including Arthrinium, Aspergillus, Myxotrichum, Nodulisporium, Lentinus, Paraphaeosphaeria, Penicillium, Pestalotiopsis, Phomopsis, Spicaria, Streptomyces, Ulocladium.

\section{Arthrinium}

One marine sponge-derived fungus Arthrinium arundinis ZSDS1-F was shown to metabolize a novel naphthalene glycoside (137) (Wang J.F. et al., 2014), five cytochalasins (138-142) (Wang et al., 2015b), and three alkaloids (143-145) when cultivated in a rotary liquid medium (Wang et al., 2015c). However, only phenethyl 5-hydroxy-4-oxohexanoate (146) was traced in rice medium (Li Y. L. et al., 2017). Bioassay suggested that compounds 143-146 possessed in vitro cytotoxicity against cancer cell lines A549, BGC823, Huh-7, K562, H1975, MCF-7, HL60, U937, Hela, and MOLT-4 with $\mathrm{IC}_{50}$ values in range of $0.24-45 \mu \mathrm{M}$. In addition, compounds 143 and 145 displayed significant AchE (acetylcholine esterase) inhibitory activity with $\mathrm{IC}_{50}$ values of 47 and $0.81 \mu \mathrm{M}$, respectively.
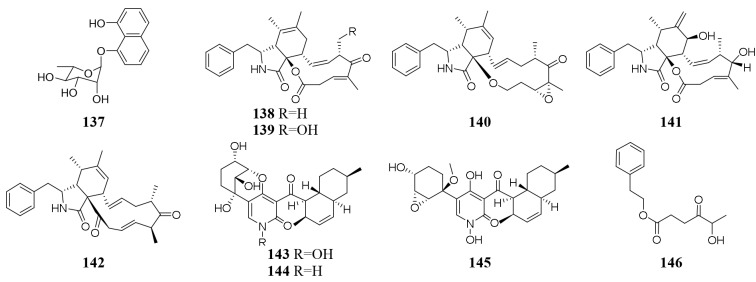

\section{Aspergillus}

By comparison of solid and liquid fermentation products of an endophytic strain A. fumigates LN-4 from stem bark of Melia azedarach L., their HPLC profiles were obviously different (Zhang et al., 2013). Strain A. versicolor ZLN-60 could produce two new cyclic pentapeptides (147-148) and four new prenylated diphenyl ethers (149-152) in static liquid condition (Zhou et al., 2011; Gao et al., 2013). Biological tests indicated that compound $\mathbf{1 5 1}$ displayed moderate cytotoxicity against Hela and $\mathrm{K} 562$ cancer cell lines with $\mathrm{IC}_{50}$ values of $31.5,48.9 \mu \mathrm{M}$, respectively. However, further purification of its crude extract of solid medium resulted in the detection of four other novel cyclic peptides (153-157) (Peng et al., 2014). Chemical study of one marine-derived fungus $A$. terreus cultivated in 11 different culture conditions indicated that static agar was ideal for the production of antifungal lovastatins (158-159) and 7-desmethylcitreoviridin (160), which were absent in the shaking fermentation (Adpressa and Loesgen, 2016).

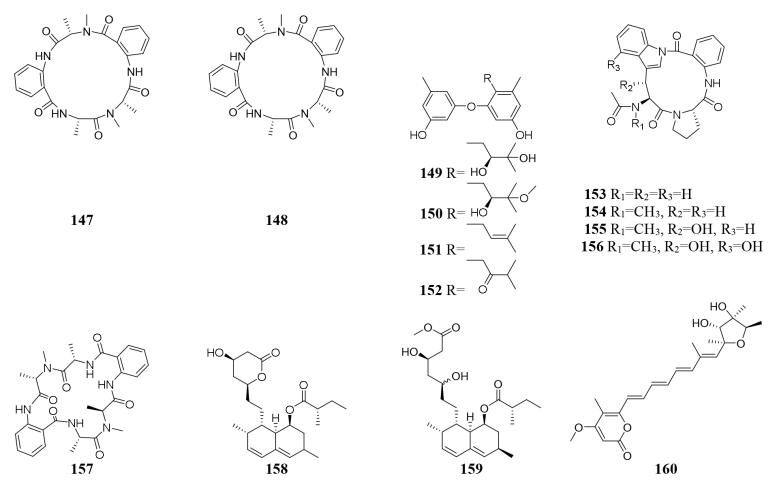

\section{Lentinus}

Two new prenyl phenols (161-162), one indole alkaloid echinuline (163) and one anthraquinone fiscione (164), were biosynthesized by Lentinus strigellus under static condition. While in shaking fermentation broth, this strain produced benzopyrans (165-168) together with panepoxydone (169) and isopanepoxydone (170). Bioassay indicated that striguellone A (171) displayed moderate cytotoxicity against HeLa cancer cells (Zheng et al., 2009; Barros et al., 2012).
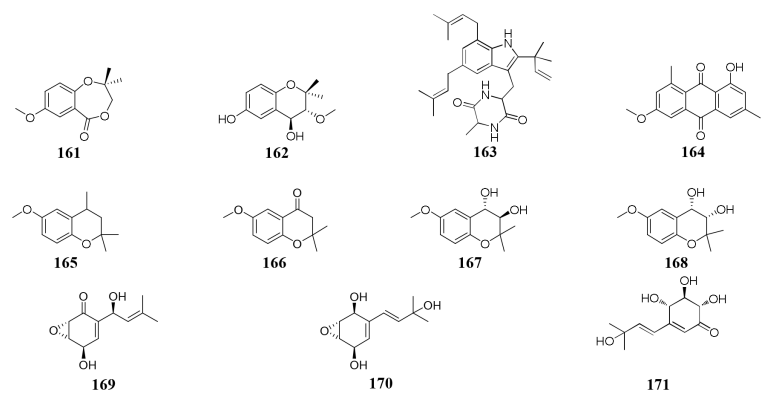

\section{Myxotrichum}

One fungal strain Myxotrichum sp. isolated from lichen Cetraria islandica (L.) Ach in Laojun Mountain (China), was shown to 
make one novel austdiol analog (172), three new fulvic acid derivatives (173-175) and a new citromycetin analog (176) in rotary PDB medium (Yuan et al., 2013), while four new polyketides (177-180) were acquired from rice medium under static fermentation status. And compound $\mathbf{1 7 9}$ was shown to restrain Arabidopsis seeds root markedly with the inhibition rate of $75.9 \%$ at $8 \mu \mathrm{g} / \mathrm{mL}$ (Yuan et al., 2016).

\section{Nodulisporium}

Chemical investigation of one symbiotic strain Nodulisporium sp. (No. 65-12-7-1) from the lichen Everniastrum sp. resulted in the isolation of two rarely 4-methyl-progesteroids (181-182) when grown in rice medium (Zheng et al., 2013). Whereas this strain could biosynthesize ten novel nodulisporisteroids (183-192) in shaking PDB medium (Zhao et al., 2015).

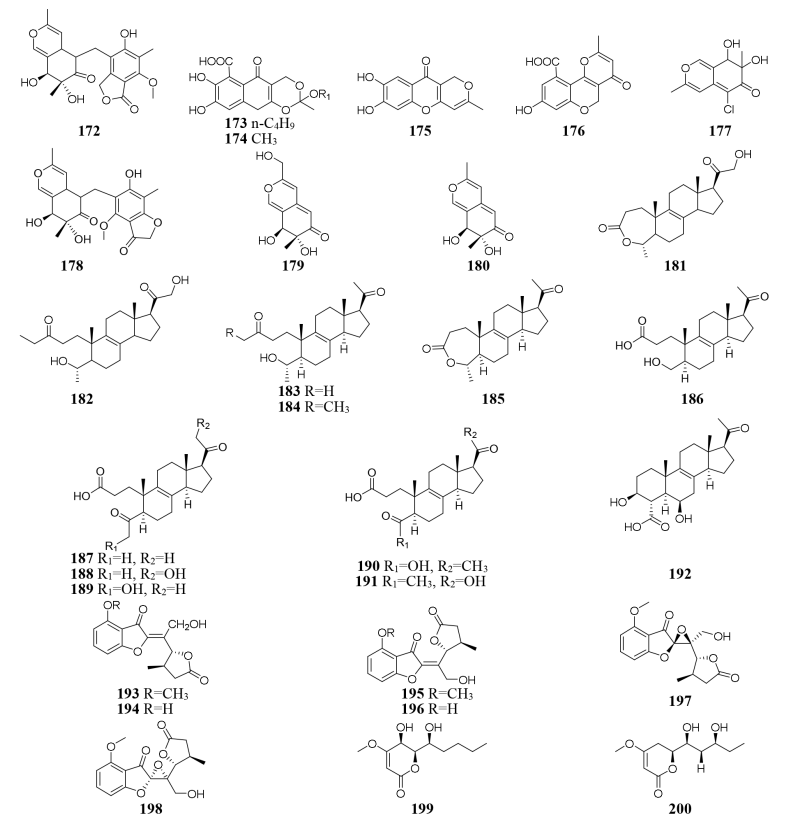

\section{Paraphaeosphaeria}

A fungal strain Paraphaeosphaeria photiniae, inhabiting Roystonea regia collected from Jianfeng Mountain (China), was shown to yield six new unique benzofuranone-derived $\gamma$-lactones (193-198) when cultivated in shaking liquid medium (Ding et al., 2009), while only two different $\delta$-lactone derivatives (199-200) were detected in its rice medium (Ding et al., 2012).

\section{Penicillium}

When grown on solid PDA medium, one mangrove-derived fungus Penicillium brocae MA-231 could produce six new disulfide-bridged diketopiperazine derivatives (201-206). Bioassay results showed that compounds 201, 202, 205, and 206 had cytotoxic activities against Du145, Hela, HepG2, MCF-7, NCI-H460, SGC-7901, SW1990, SW480, and U251 tumor cell lines with $\mathrm{IC}_{50}$ values ranging from 0.89 to $9.0 \mu \mathrm{M}$ (Meng et al., 2014). When cultivated in liquid media (PDB or Czapek), however, five new penicibrocazines (207-211), four new thiodiketopiperazine alkaloids (212-215) and two new
$N$-containing $p$-hydroxyphenopyrrozin derivatives (216-217) were detected in its fresh mycelia, which compounds 207-209 displayed antimicrobial activities against Staphylococcus aureus with MIC values of $32.0,0.25,8.0 \mu \mathrm{g} / \mathrm{mL}$, respectively. In addition, 209-211 exhibited potent antimicrobial effect on Gaeumannomyces graminis with MIC values of $0.25,8.0$ and $0.25 \mu \mathrm{g} / \mathrm{mL}$, respectively. And compound 216 showed powerful inhibitory effect on Fusarium oxysporum and S. aureus (Meng et al., 2015b, 2017).

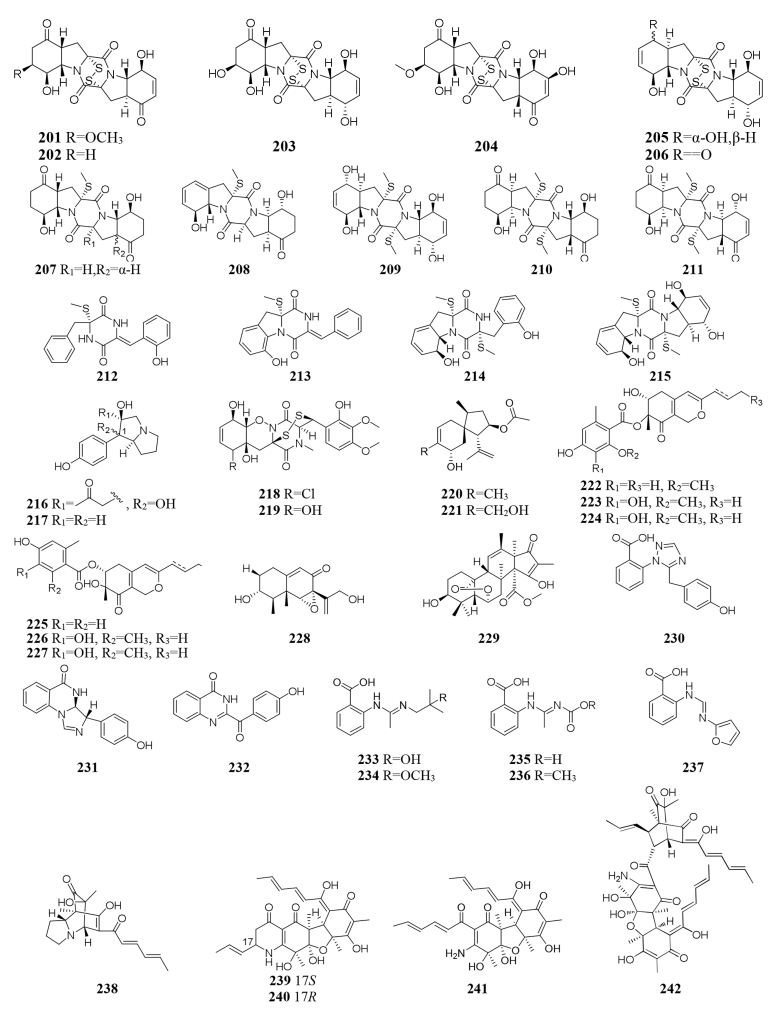

Chemical study of one marine sponge-derived strain $P$. adametzioides AS-53 led to isolation of two new bisthiodiketopiperazine derivatives (218-219) from shaking PDB broth, whereas two new acorane sesquiterpenes (220-221) were found in its static rice medium. Compound 218 showed strong lethality against brine shrimp (Artemia salina) with an $\mathrm{LD}_{50}$ value of $4.8 \mu \mathrm{M}$ and a broad spectrum of antimicrobial effect on Aeromonas hydrophilia, S. aureus, Vibrio spp. V. harveyi, Gaeumannomyces graminis and $V$. parahaemolyticus (Liu Y. et al., 2015). Six novel azaphilone derivatives (222-227) as major secondary metabolites were obtained from rotary PDB medium of one marine-derived strain P. commune QSD-17 (Gao et al., 2011), whereas other new compounds isophomenone (228) and 3-deacetylcitreohybridonol (229) were detected in its static rice medium (Gao et al., 2012).

Three novel penipanoids (230-232) were characterized from one marine-derived strain $P$. paneum SD-44 grown in rice medium ( $\mathrm{Li}$ et al., 2011). The exploration of changing fermentation conditions of $P$. paneum SD-44 to a seawater-based culture broth under dynamic fermentation condition gave five 
new anthranilic acid derivatives (233-237). Metabolites 233 and 237 exhibited inhibitory activity toward human colon cancer RKO cell lines with $\mathrm{IC}_{50}$ values of $8.4,9.7 \mu \mathrm{M}$, respectively (Li C. S. et al., 2013). One deep sea-derived fungus Penicillium sp. F23-2 biosynthesized terpenoids, diketopiperazines, and meleagrin alkaloids when incubated in sea-water-based culture medium under static condition (Du et al., 2009, 2010), whereas five new nitrogen-containing sorbicillinoids (238-242) were metabolized by this stain when cultivated in PYG (peptone yeast glucose) medium under shaking status (Guo et al., 2013).

\section{Pestalotiopsis}

When grown in rice medium, one endophytic strain of Pestalotiopsis fici from Camellia sinensis was found to be a prolific producer of bioactive secondary metabolites, including pupukeanane chloride (243) (Liu et al., 2008a), chloropestolide A (244) (Liu et al., 2009a), seven isoprenylated chromones (245-251) (Liu et al., 2010), three highly functionalized compounds (252-254) (Liu et al., 2009b), and three cytotoxic pupukeanane chlorides (255-257) (Liu et al., 2011). In vitro cytotoxic assays suggested that compound 244 possessed potent inhibitory effects on HeLa and HT29 with $\mathrm{GI}_{50}$ values of $0.7,4.2 \mu \mathrm{M}$, respectively. However, this strain produced new cyclopropane derivatives (258-262) when cultivated in shaking liquid medium (Liu et al., 2008b). An endophytic fungus $P$. foedan, residing in Bruguiera sexangul, synthesized a new reduced spiro azaphilone derivative (263) together with two new isobenzofuranones (264-265) in solid GYM (glucose, yeast extract, malt) medium (Ding et al., 2008). But, in liquid modified PDB medium, a pair of novel spiro- $\gamma$-lactone enantiomers (266-267) were identified (Yang and Li, 2013).

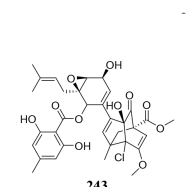

243
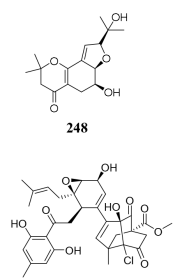

254

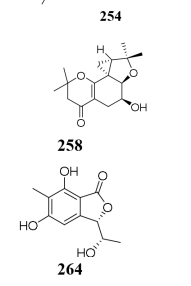

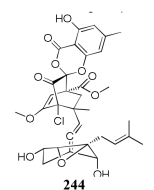
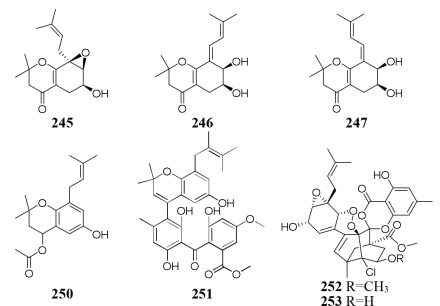

251
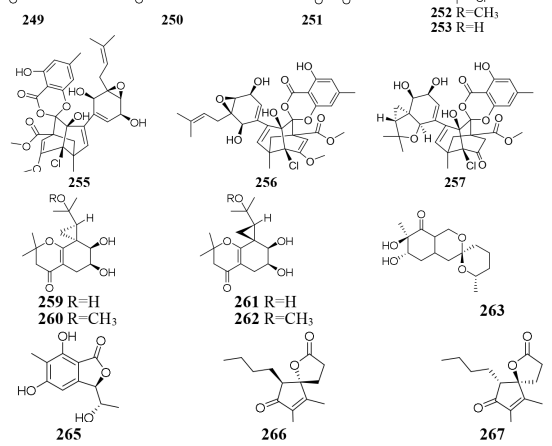

267

\section{Phomopsis}

An endophytic fungus Phomopsis sp. sh917 isolated from fresh stems of Isodon eriocalyx var. laxiflora collected in Kunming Botanical Garden of China, was shown to produce six new polyketides (268-273) on solid rice medium but metabolize a new polyketide (274) in shaking liquid FM4 medium (Tang et al., 2017).

\section{Spicaria}

Nine new cytochalasins Z7-Z15 (275-283), one novel spicochalasin (284), five new aspochalasins (285-289), and three new aspochalasin derivatives (290-292) were synthesized by a marine-derived fungus Spicaria elegans KLA03 in the seawater-based medium under static fermentation status. Compounds 235 and 276 displayed strong cytotoxicity against P388 and A-549 cancer cell lines with $\mathrm{IC}_{50}$ values in range of 8.4-99 4M (Liu et al., 2005, 2006, 2008c; Lin et al., 2009a, 2010). However, new aromatic polyketide (293) was obtained from shaking seawater medium (Luan et al., 2014).

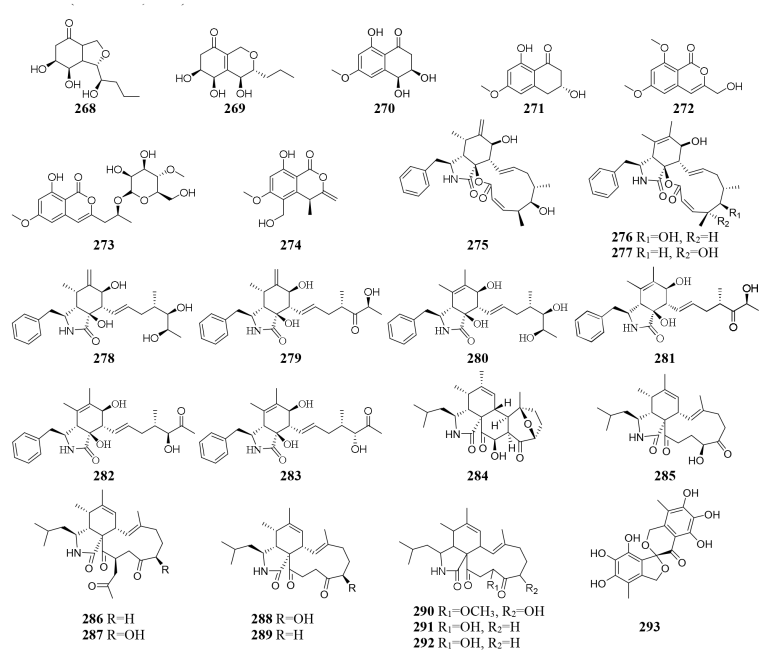

\section{Streptomyces}

One marine-derived stain Streptomyces sp. CHQ-64 was found to produce six new antifungal polyene-polyols (294-299) and two new cytotoxic hybrid isoprenoid alkaloids (300-301) in liquid medium under shaking condition, while this strain made only one new hybrid isoprenoid alkaloid (302) under static condition (Che et al., 2012, 2013, 2015, 2016). When cultivated in liquid Gause's No. 1 medium, strain Streptomyces sp. DT-A37 could produce a new ring-opened lactam (303), while in rice medium one unknown holomycin (304) and two new cyclopropaneacetic acids (305-306) were detected (Ding et al., 2017). Strain Streptomyces sp. HZP-2216E cultured in 2216E solid medium, GYM solid medium and GMSS (Gause's medium with sea salt) liquid medium resulted in isolation of two new compounds of 23-O-butyrylbafilomycin D (307), streptoarylpyrazinone A (308) a unique indolizinium alkaloid streptopertusacin A (309). It was noted that compound $\mathbf{3 0 7}$ showed potent activity in suppressing the proliferation of the four tested glioma cell lines with $\mathrm{IC}_{50}$ values in a range from 0.35 to $2.95 \mu \mathrm{M}$ and antibacterial activity with MIC value of $7.4 \mu \mathrm{M}$ for MRSA and $\mathrm{IC}_{50}$ values of 0.44 to $0.98 \mu \mathrm{M}$ for glioma cells (Zhang et al., 2017c,d). 


\section{Ulocladium}

Two antifungal polyketides (310-311) were characterized from rice medium of Ulocladium $\mathrm{sp}$. that was isolated from the lichen Everniastrum sp. (Wang X.E. et al., 2012), whereas three new tricycloalternarenes F-H (312-314) and five ophiobolane sesterterpenes (315-319) were detected in liquid Czapek or PDB medium (Wang et al., 2013a,b). Compounds 315 and 319 exhibited moderate antibacterial activity against meticillinresistant S. aureus and Bacillus subtilis and displayed strong in vitro cytotoxicity against cancer cell lines $\mathrm{KB}$ and HepG2.

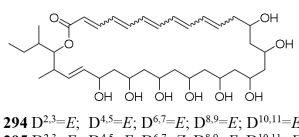

$294 \mathrm{D}^{2,3}=E ; \mathrm{D}^{4,5}=E ; \mathrm{D}^{6,7}=E ; \mathrm{D}^{8,9}=E ; \mathrm{D}^{10,11}=E$ $296 \mathrm{D}^{2,3}=Z ; \mathrm{D}^{4,5}=E ; \mathrm{D}^{6,7}=E ; \mathrm{D}^{8,9}=E ; \mathrm{D}^{10,11}=E$ $2967 \mathrm{D}^{2,3}=Z ; \mathrm{D}^{4,3}=E ; \mathrm{D}^{4,5}=E ; \mathrm{D}^{6,7}=E ; \mathrm{D}^{8,9}=E ; \mathrm{D}^{8,9}=E ; \mathrm{D}^{10,11}=Z$
29.11 $298 \mathrm{D}^{2,3}=\mathrm{E} ; \mathrm{D}^{4,5}=Z ; \mathrm{D}^{6,7}=E ; \mathrm{D}^{8,9}=Z ; \mathrm{D}^{10,11}=E$

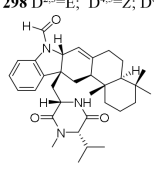

300
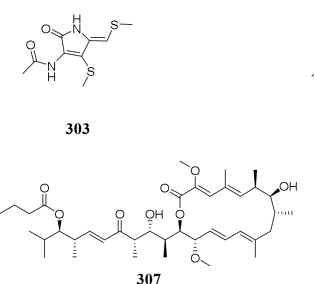

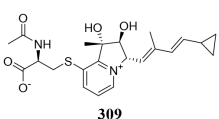

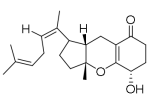

312

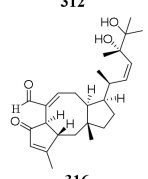

316

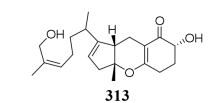
313
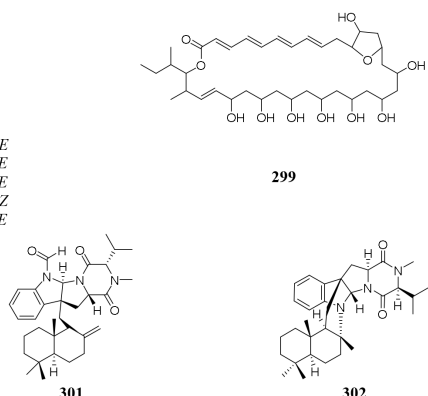

299

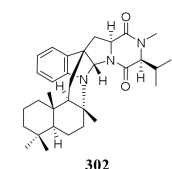

302

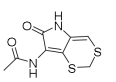

304
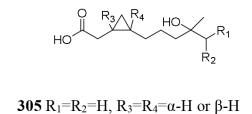
$305 \mathrm{R}_{1}=\mathrm{R}_{2}=\mathrm{H}, \mathrm{R}_{3}=\mathrm{R}_{4}=\alpha-\mathrm{H}$ or $\beta-\mathrm{H}$
$306 \mathrm{R}_{4}=\mathrm{CH}_{3}, \mathrm{R}_{2}=\mathrm{OH}, \mathrm{R}_{3}=\mathrm{R}_{4}=\alpha-\mathrm{H}$ or $\beta-\mathrm{H}$ (

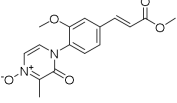

308
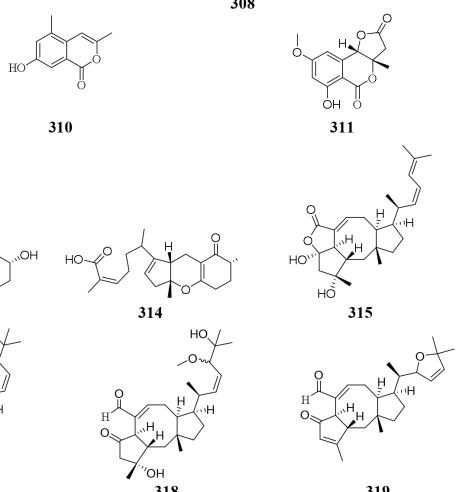

317

319

\section{CO-CULTIVATION WITH OTHER STRAIN(S)}

In one culture medium, the relationship between one strain and other(s) may be competitive, antagonistic or friendly. Co-cultivation of two or more strains usually has positive effect of an enhanced production of known compounds or an accumulation of cryptic compounds that are not detected in axenic culture (Bohni et al., 2013; Marmann et al., 2014). This effect maybe results from the production of enzymes that activate metabolite precursors or that other strain(s) may induce epigenetic modifications of the producer strain.

\section{Fungus and Other Fungal Strain}

An endophytic strain Acremonium sp. Tbp-5 from the European yew (Taxus baccata L.) could produce new lipoaminopeptides (320-322) when co-cultivated with Mycogone rosea DSM 12973 (Degenkolb et al., 2002). Chemical investigation of the mixed fermentation broth of two epiphytic strains Aspergillus sp. FSY-01 and FSW-02 from marine mangrove Avicennia marina led to the isolation of a novel alkaloid (323), which had antibacterial activity against Bacillus dysenteriae, B. proteus, and E. coli (Zhu et al., 2011). The production of 2-alkenyl-tetrahydropyran analogs (224-326) was provoked by Chaunopycnis sp. CMB-MF028 in the mixed culture with a partner strain Trichoderma hamatum CMB-MF030, which were isolated from the inner tissue of marine pulmonate false limpet (Shang et al., 2017). Co-cultivation of Monascus sp. J101, used as the producer of Monascus pigment, with Saccharomyces cerevisiae KCCM 11371 or A. oryzae KCCM 11773 on the solid sucrose medium could result in two folds of accelerated cell growth and 30-40 folds of increased pigment production (Shin et al., 1998). Strain J101 was shown to stimulate cell growth and reproduction by interacting with S. cerevisiae, which resulted in production of more hydrophobic pigments compared to the mono-culture (Suh and Shin, 2000a,b). When co-cultivated with Beauveria felina, one marine-derived P. citrinum could biosynthesize two new compounds (327-328) featuring in a unique tetracyclic framework, whereas neither strain could produce these compounds in axenic medium. Antimicrobial assay showed that compounds $\mathbf{3 2 7}$ and $\mathbf{3 2 8}$ had strong inhibitory effects on human pathogens $S$. aureus and E. coli (Meng et al., 2015a). Penicillium sp. IO1 derived from mediterranean sponge Ircinia oros could produce a new fusarielin analog (329). However, co-cultivation of Penicillium strains IO1 and IO2 resulted in the accumulation of two known compounds norlichexanthone and monocerin, which were not detected in axenic controls (Chen et al., 2015a). Four new polyketides (330-333) were detected in a dual culture of the deep-sea-derived fungus Talaromyces aculeatus and a mangrove-derived fungus

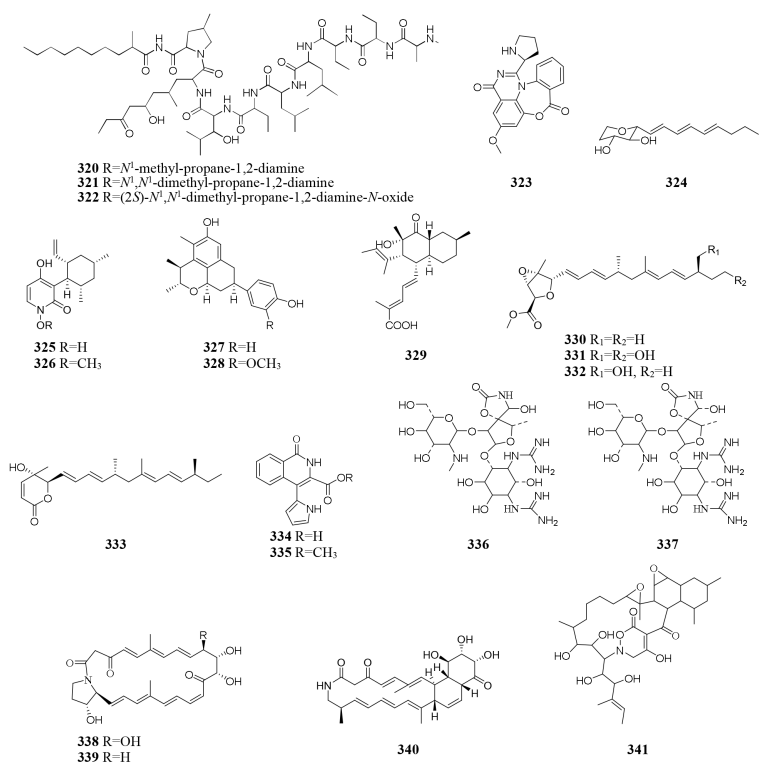


$P$. variabile, while these compounds were not identified in single culture. Compounds $\mathbf{3 3 3}$ displayed strong cytotoxicity against A549, K562, HCT-116, HeLa, MCF-7 and HL-60 human cancer cell lines with $\mathrm{IC}_{50}$ values ranging from 1.2 to $9.8 \mu \mathrm{M}$ (Zhang et al., 2017b).

One novel 1-isoquinolone analog (334) and its methyl ester (335) were detected in mycelia and culture filtrate of mixed fermentation of two endophytic fungi Nos. $1924^{\#}$ and $3893^{\#}$, whereas these compounds were not traced in axenic medium under the same conditions (Zhu and Lin, 2006). The formation of new antibiotics (336-337) was emerged during co-cultivation of a multi-antibiotic stable mutant strain of Rhodococcus fascians and a strain Streptomyces padanus, neither of which was capable of yielding an antibiotic (Kurosawa et al., 2008). An terrestrial bacterium Tsukamurella pulmonis TP-B0596 co-cultured with strain Streptomyces sp. NZ-6, coincided with stimulation of three new metabolites (338-340) of unprecedented di-andtricyclic macrolactams (Hoshino et al., 2015). The yield of red pigment was detected in the dual induction of T. pulmonis TP-B0596 and $S$. lividans TK23. Co-cultivation of T. pulmonis and S. endus S-522 resulted in the production of one new antibiotics called alchivemycin A (341) (Onaka et al., 2011). A soil-dwelling actinomycetes $S$. coelicolor was shown to significantly improve the yield of red compound undecylprodigiosin, when co-cultured with Corallococcus coralloides (Schäberle et al., 2014).

\section{Bacterium and Other Bacterial Strain}

Only two new macrolactams (342-343) were detected in the co-culture broth of a rare actinomycete Micromonospora wenchangensis HEK-797 and Tsukamurella pulmonis TPB0596, whereas the axenic medium of strain HEK-797 produced a polyene macrolactam (344), which was possibly the precursor of compounds 342 and 343 (Hoshino et al., 2017). Investigation of the interaction of the portable predator Myxococcus Xanthus and Streptomyces coelicolor showed that actinorhodin production of $S$. coelicolor was raised up to 20-fold and stimulated aerial mycelium production (Pérez et al., 2011). Co-cultivation of two sponge-derived actinomycetes, Nocardiopsis sp. RV163 and Actinokineospora sp. EG49, induced ten reported compounds, including diketopiperazine, angucycline, and $\beta$-carboline derivatives, while only three natural products were isolated in mono-culture (Dashti et al., 2014). Mixed culture of Pseudomonas maltophilia 1928 and S. griseorubiginosus 43708 resulted in the production of one peptide antibiotic, biphenomycin A (345) (Ezaki et al., 1992). However, the accumulation of biphenomycin A, which could be obtained from the transformation of biphenomycin C (346), was inhibited in single culture of strain 1928 (Uchida et al., 1985; Ezaki et al., 1993). Interspecies interactions between Streptomyces coelicolor M145 with other actinomycete stains (Amycolatopsis sp. AA4, Streptomyces sp. E14, Streptomyces sp., SPB74 and S. viridochromogenes DSM 40736) resulted in the production of at least 12 different versions of a molecule called desferrioxamine (Traxler et al., 2013).

\section{Fungus and Bacterium}

Co-cultivation of one fungal strain $A$. terreus with $B$. cereus and $B$. subtilis resulted in the yield of two novel butyrolactones
(347-348), which were absent in single culture medium (Chen et al., 2015b). An endophyte Chaetomium sp. from the Cameroonian plant Sapium Ellipticum (Euphorbiaceae) was shown to produce two novel shikimic acid analogs (349-350) and four new butenolide derivatives (351-354) when co-cultivated with Pseudomonas aeruginosa, while none of these chemicals was traced in axenic medium (Ancheeva et al., 2017). Strain Bacillus subtilis 168 trpC2 was shown to greatly activate the biosynthesis of three novel chemicals (355-357) of fungal endophyte Fusarium tricinctum during co-culture process. And these compounds were not duplicated in axenic fungal culture (Ola et al., 2013).

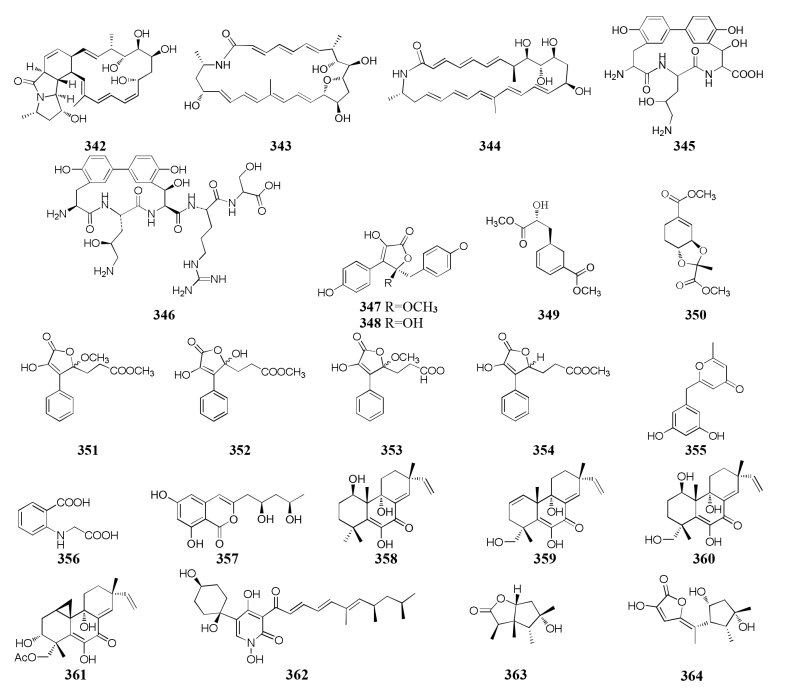

Co-cultivation of one marine fungus Libertella sp. CNL523 symbiotic on an ascidian collected from the Bahamas and a fellow strain Thalassospira sp. CNJ-328 resulted in the production of four new diterpenoids (358-361). Compound 360 exhibited remarkable cytotoxicity against HCT-116 human adenocarcinoma cell line with an $\mathrm{IC}_{50}$ value of $0.76 \mu \mathrm{M}(\mathrm{Oh}$ et al., 2005). A new pyridone alkaloid (362) was isolated from the mixed culture extract of Paecilomyces lilacinus and Salmonella typhimurium, which had $57.5 \pm 5.50 \%$ of AChE inhibition (Teles and Takahashi, 2013). Co-culture of an endophyte Pestalotiopsis sp. from Drepanocarpus lunatus with B. subtilis was found to biosynthesize two novel sesquiterpenoids (363-364) while new compounds 365 and 366 emerged in axenic culture (Liu et al., 2017). The mixed cultivation of Trichoderma sp. 307 colonizing in Clerodendrum inerme and one bacterium Acinetobacter johnsonii B2 led to the production of two new sesquiterpenes (367-368) and three novel de-O-methyl lasiodiplodins (369-371). Compounds 369 and 370 displayed potent $\alpha$-glucosidase inhibitory effect with $\mathrm{IC}_{50}$ values of 25.8 and $54.6 \mu \mathrm{M}$, respectively (Zhang et al., 2017a).

Chemical study of an endophytic stain Aspergillus austroafricanus from Eichhornia crassipes led to the isolation of a highly oxygenated heterodimeric xanthone (372) and a new sesquiterpene (373) in axenic culture. Mixed fermentation of A. austroafricanus with B. subtilis or S. lividans afforded several 
diphenyl ethers, including one new austramide (374) (Ebrahim et al., 2016). Two novel $N$-formyl alkaloids (375-376) were characterized from a mixed fermentation of $A$. fumigatus and S. peucetius. Compound $\mathbf{3 7 6}$ displayed in vitro cytotoxic effect on cancer cell line NCI-60 with an $\mathrm{IC}_{50}$ value of $1.12 \mu \mathrm{M}$ (Zuck et al., 2011). Seven known diketopiperazine alkaloids associated with ergosterol and 11-O-methylpseurotin A were traced in response to the supplement of A. fumigatus MBC-F1-10 to an established culture of $S$. bullii, whereas neither strain metabolized these compounds when cultivated alone (Rateb et al., 2013). Co-cultivation of A. fumigatus MR2012 with S. leeuwenhoekii C34 in ISP2 medium resulted in the yield of a new luteoride derivative (377) and a new pseurotin derivative (378). None of these compounds could be detected in axenic culture. When strain MR2012 was co-cultivated with strain C58, a lasso peptide chaxapeptin (379) was made, which displayed significant inhibitory effect on human lung cancer cell line A549 (Elsayed et al., 2015; Wakefield et al., 2017).

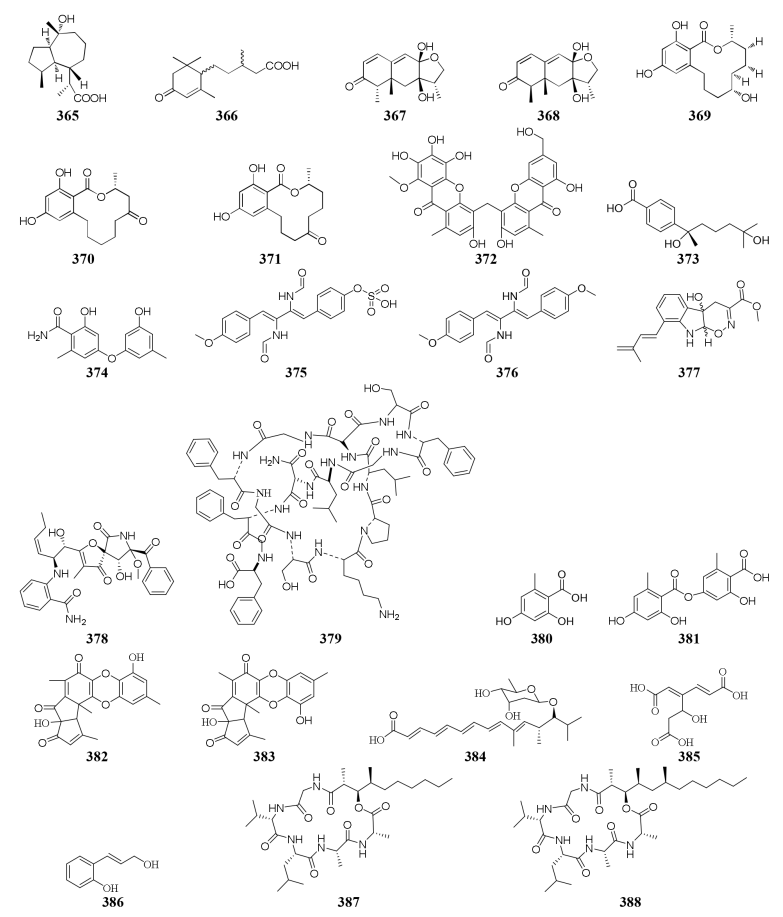

Physical interaction of $A$. nidulans RMS011 with S. hygroscopicus was found to trigger biosynthesis of four new aromatic polyketides (380-383), which were absent in the axenic medium (Schroeckh et al., 2009). A new polyketide glycoside (384) was formed in the dual induction of two Gram-positive bacteria, S. tendae KMC006 and Gordonia sp. KMC005, which were obtained from an acidic mine drainage sample (Park et al., 2017). In response to S. coelicolor A3(2) M145, strain A. niger N402 was shown to be apt to produce 2-hydroxyphenylacetic acid and cyclic dipeptide cyclo(Phe-Phe). Biotransformation of a new hexadienedioxic acid (385) and a new phenol derivative (386) was achieved by co-culture of these strains ( $\mathrm{Wu}$ et al.,
2015). More interestingly, co-cultivation of one marinederived fungus Emericella sp. CNL-878 with Salinispora arenicola $\mathrm{CNH}-665$ resulted in the higher yields of two novel antimicrobial cyclic depsipeptides (387-388) than axenic culture (Oh et al., 2007).

\section{EPIGENETIC MODIFIER}

Epigenetic modifiers are those chemicals that are able to change microbial characteristics in correspondence to alteration of their epigenetic status, such as DNA methyltransferase (DNMT) inhibitor and histone deacetylase (HDAC) inhibitor. The addition of these modifiers usually suppresses the activity of related enzymes in the biosynthetic pathway and promotes the progress of other metabolic pathways (Seyedsayamdost, 2014).

\section{DNA Methyltransferase Inhibitor}

DNA methylation is a process by which methyl groups are added to DNA. When located in a gene promoter, DNA methylation typically acts to repress gene transcription and causes chromatin structure changes in the corresponding regions, preventing the binding of specific transcription factors and suppressing gene expression (Araujo et al., 2001). 5-Azacytidine (5-AC) is the most common DNMT inhibitor used to modify the function of microbe DNA followed by repressing gene transcription. Chemical investigation of a marine-derived fungus Aspergillus sydowii afforded three novel bisabolane-type sesquiterpenoids (389-391) when its culture medium was supplemented with 5-AC (Chung et al., 2013b). An entomopathogenic fungus Cordyceps indigotica yielded a novel aromatic polyketide

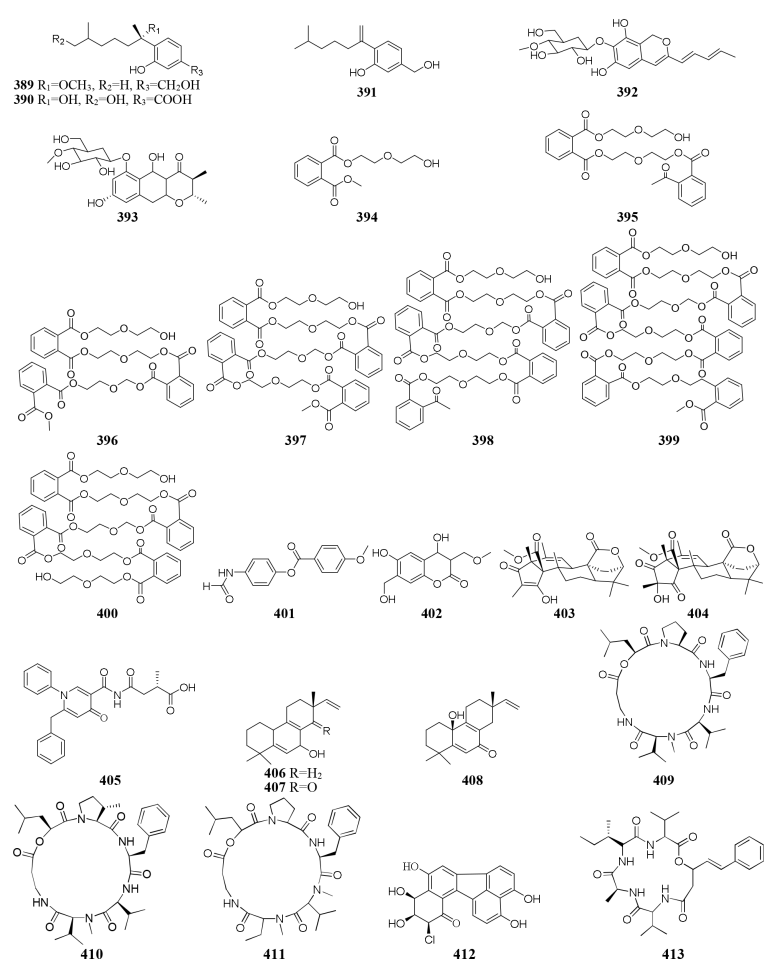


glycoside (392) when cultivated in PDB media, while the strain produced another unusual glycoside (393) when supplement with 5-AC (Asai et al., 2012e). Several other examples that adding $5-\mathrm{AC}$ as epigenetic modifier in culture medium could lead to the production of new metabolites were also reported, such as novel diethylene glycol phthalate esters (394-400) from a marine-derived strain Cochliobolus lunatus TA26-46 (Chen et al., 2016), a new benzoic acid (401) from Pestalotiopsis microspora (Yang et al., 2017), one new coumarin (402) from P. crassiuscula NBRC 31055 associated with Fragaria chiloensis (Yang et al., 2014), and novel meroterpenes (403-404) from P. citreonigrum (Wang et al., 2010).

\section{Histone Deacetylase Inhibitor}

The acetylation or deacetylation of histone affects its binding to DNA in microbe. There are many chemical modifications in the tail of histone that regulate the gene expression. The introduction of hydrophobic acetyl group into the $\mathrm{N}$-terminal lysine residues of histone could increase the electrostatic attraction and steric hindrance between histone and DNA, which is conducive to facilitate the depolymerization of DNA and the binding of transcription factors (Fukuda et al., 2006; Cole, 2008). Suberoyl bishydroxamic acid (SBHA), suberoylanilide hydroxamic acid (SAHA), and nicotinamide are the most common HDAC chemicals used to inhibit the deacetylation and facilitate gene transcription and expression in microbes (Moore et al., 2012).

Many reports suggested the presence of SAHA in culture medium could result in production of new natural compounds, such as a novel metabolite nygerone A (405) from a soildwelling fungus A. niger ATCC 1015 (Henrikson et al., 2009), two new aromatic norditerpenes (406-407) tied with an oxygenated derivative (408) from a marine-derived $A$. wentii na-3 residing in the brown alga Sargassum fusiforme (Miao et al., 2014), three novel cyclodepsipeptides (409-411) from Beauveria feline (Chung et al., 2013a), one novel chlorinated polyketide (412) from Daldinia sp. (Du et al., 2014), a new cyclodepsipeptide of hybrid EGM-556 (413) from one marine sediment-derived fungus Microascus sp. (Vervoort et al., 2011).

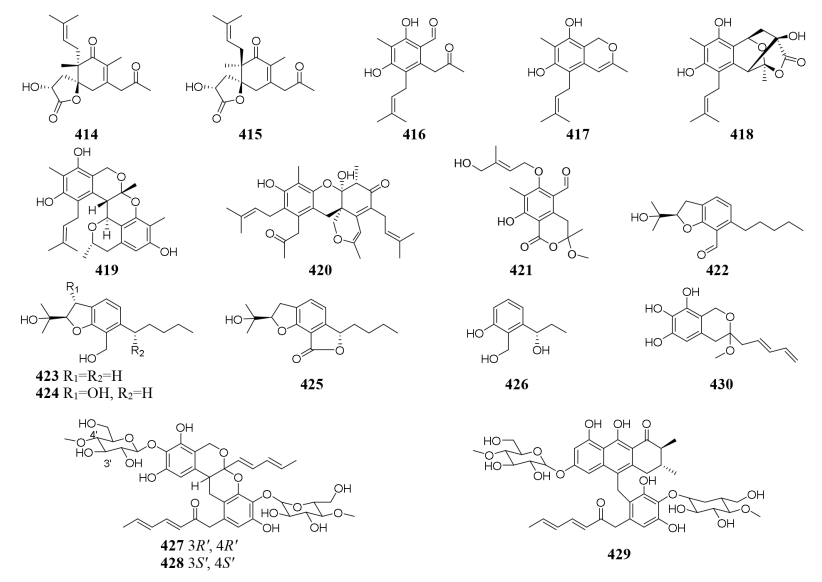

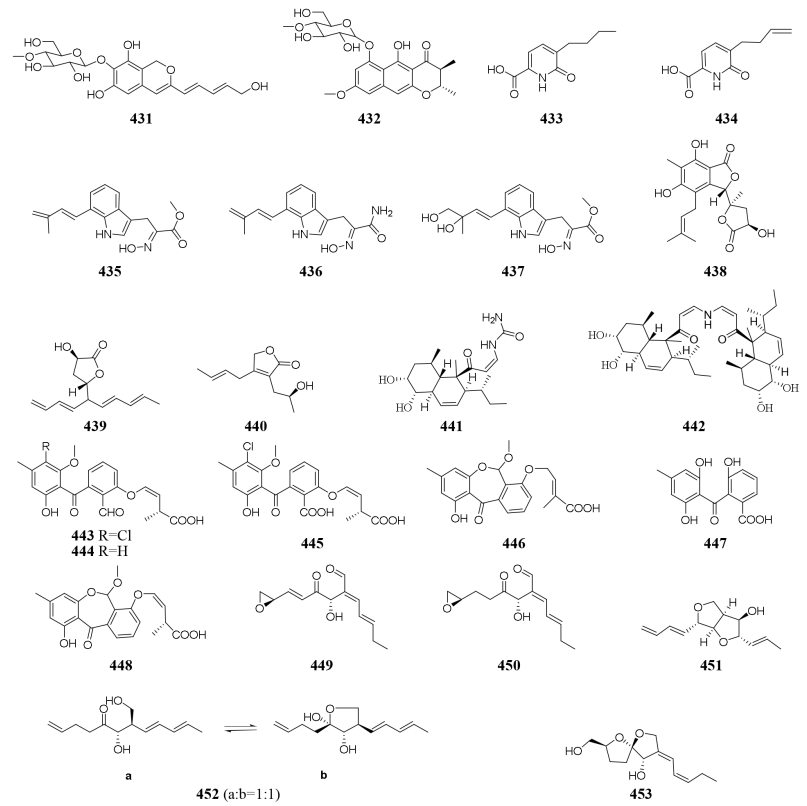

In SBHA-treated culture medium, Chaetomium indicum could produce two novel spironolactone polyketides (414-415) and six novel prenylated aromatic polyketides (416-421) (Asai et al., 2013b,c). Similarly, when exposed to SBHA, four new 2,3-dihydrobenzofurans (422-425) and a new aromatic polyketide (426) were characterized from an entomopathogenic fungus Cordyceps annullata (Asai et al., 2012c), six new aromatic polyketides (427-432) were synthesized by C. indigotica (Asai et al., 2012f), two new fusaric acid derivatives (433-434) were produced by Fusarium oxysporum associated with medicinal plant Datura stramonium L. (Chen et al., 2013), and a series of novel prenylated tryptophan analogs (435-437) were metabolized by an entomopathogenic fungus Torrubiella luteorostrata (Asai et al., 2011). Supplement of nicotinamide [a $\mathrm{Zn}$ (II)-type HDAC inhibitor] in culture medium of C. cancroideum could generate three novel polyketides (438-440) (Asai et al., 2016). The use of this inhibitor to strains Eupenicillium sp. LG41 and Graphiopsis chlorocephala had the similar effect, which the former supplied two new decalin-containing compounds (441-442) (Li G. et al., 2017) and the later afforded a serious of new benzophenones (443-444) and diverse new $\mathrm{C}^{13}$-polyketides (445-453) (Asai et al., 2012d, 2013a).

\section{Multiple Chemical Epigenetic Modifiers}

Interactions between epigenetic features play an important role in regulation of gene expressing or silencing in microorganisms, such as DNA methylation and histone modification. Many references that looked into the combined effect of epigenetic processes suggested that these chemicals could regulate the activity of genomic regions of varying sizes, from single genes to entire domains and chromosomes. Epigenetic markers could also interact with other nuclear proteins to work together to form chromatin structures and to create genomic functional discrete 
regions that induce the production of new secondary metabolites (Tammen et al., 2013).

One symbiotic strain Alternaria sp. from medicinal plant Datura stramonium Linn. was shown to produce four new aromatic polyketides (454-457) and a new tenuazonic acid (458) when incubated in medium containing 5-AC and/or SBHA. While these compounds were absent in normal culture medium. Interestingly, the yield of these secondary metabolites was higher in the medium of adding HDAC and DNMT inhibitors than that of addition of any other inhibitors (Sun et al., 2012). Chemical investigation of one marine-derived fungus Aspergillus sp. SCSIOW2 or SCSIOW3, exposed with an integration of SHBA and $5-\mathrm{AC}$, led to production of three new eremophilane-type sesquiterpenes (459-461) together with a new diphenylether- $O$ glycoside (462) (Wang L. et al., 2016; Li X. et al., 2017). Bioactivity tests indicated that the glycosylated compound 462 exhibited a protective activity toward free radicals with an $\mathrm{EC}_{50}$ value of 20.8 $\mu \mathrm{M}$. One strain Cladosporium cladosporioides from a tidal pool was found to display different responses to the treatment with 5$\mathrm{AC}$ and SHBA. Exposure of C. cladosporioides to 5-AC resulted in substantially increased biosynthesis of three oxylipins (463-465), whereas SHBA induced the yield of two new perylenequinones (466-467) (Williams et al., 2008).

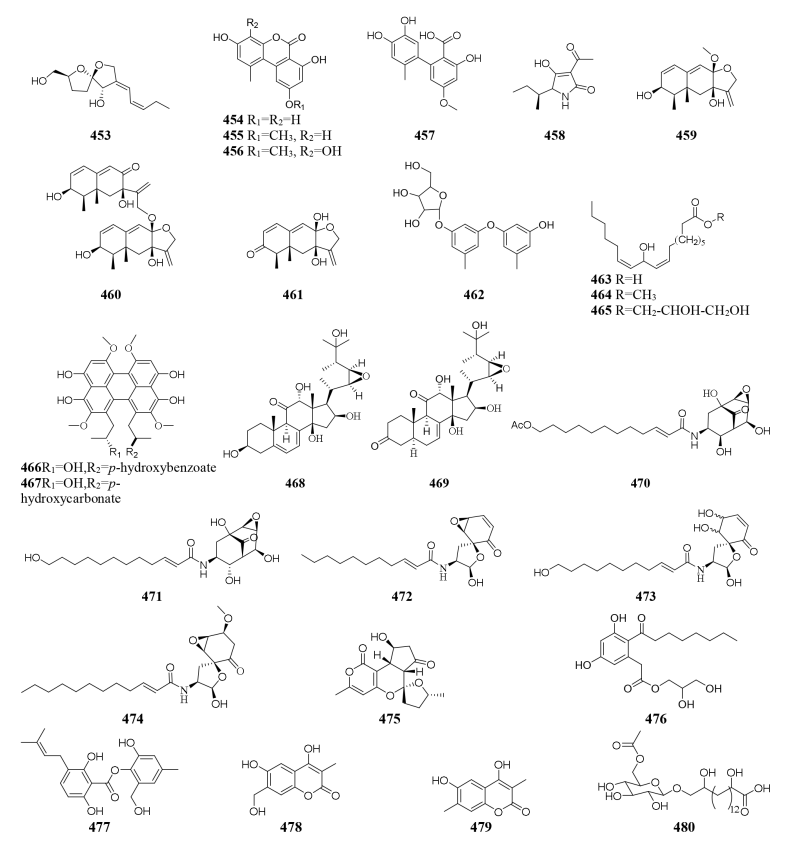

Concomitant supplement of SHBA and $N$-phthalyl- $L$ tryptophan (DNMT inhibitor) to the fermentation medium of an entomopathogenic fungus Gibellula formosana induced the formation of two new highly oxidized ergosterols (468-469) and five new isariotin analogs (470-474) (Asai et al., 2012a). The same method was applied to expand MSM profile of Isaria tenuipes, which resulted in the yield of one new polyketide (475) (Asai et al., 2012b). An endophytic strain Leucostoma persoonii from red mangrove was subject to large-scale cultivation with sodium butyrate (HDAC inhibitor) and 5-AC, which resulted in the increased yield of known cytosporones and the production of one new cytosporone (476) (Beau et al., 2012). Three novel aromatics (477-479) were produced by Pestalotiopsis acacia from Taxus brevifolia when its culture medium was supplemented with SHBA and 5-AC (Yang and Li, 2013). Application of this approach also led to production of a new glycolipid ustilagic acid C (480) by Ustilago maydis (Yang et al., 2013).

\section{OTHER FACTORS}

\section{Enzyme Inhibitor}

Beside DNMT and HDAC, other microbial enzymes also played important role in the regulating the biosynthesis of secondary metabolites, such as monooxygenase and hydroxylase. Some chemicals can selectively inhibit the activity of these enzymes in the biosynthetic pathway and promote the progress of other metabolic pathways, such as metyrapone, tricyclazole, jasplakinolide, and DMSO.

Chemical study of Chaetomium subaffine in the presence of metyrapone (an inhibitor of cytochrome P-450) led to purification of five new polyketides (481-485) and two new less oxidized analogs (486-487) (Oikawa et al., 1992). A soilderived strain Phoma sp. SNF-1778 was shown to yield a new cytochalasin (488) when inoculated with metyrapone (Kakeya et al., 1997). When added with the F-actin inhibitor jasplakinolide in culture medium, one marine sponge-derived fungus Phomopsis asparagi could afford three unusual cytotoxic compounds, chaetoglobosin-510 (489), chaetoglobosin-540 (490), and chaetoglobosin-542 (491) (Christian et al., 2005). Two novel bisnaphthalene compounds (492-493) were characterized from Sphaeropsidales sp. F-24'707 cultured with tricyclazole, which was shown to inhibited the regular biosynthesis of 1,8dihydroxynaphthalene (Bode and Zeeck, 2000). Continuous study showed that metyrapone supplementation in the culture of Spicaria elegans led to the isolation of two novel 7-deoxy-cytochalasins (494-495). Compound 494 had weak

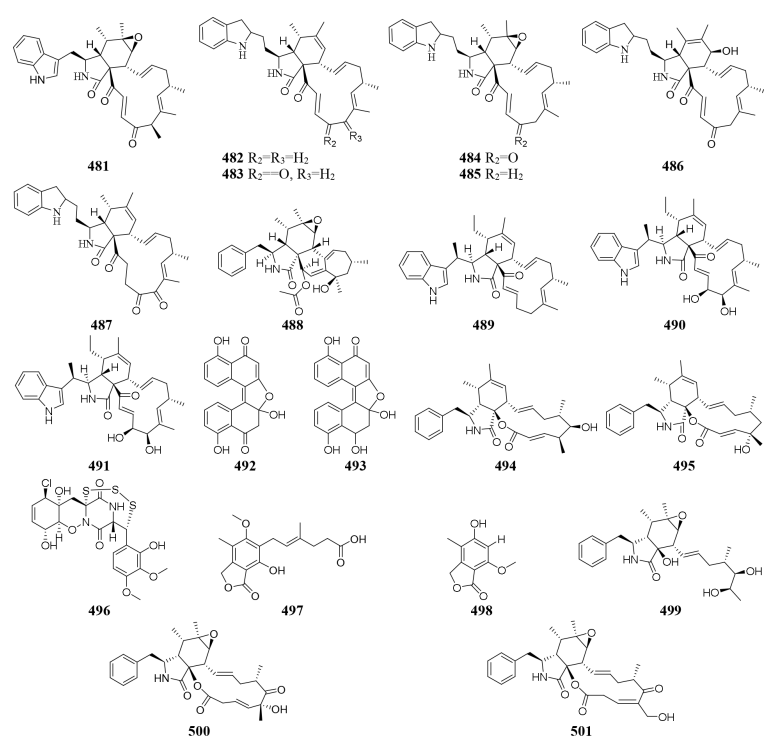


cytotoxicity against human lung cancer cell line A-549 at $15.0 \mathrm{mM}$ (Lin et al., 2009b). One marine-derived strain Trichoderma cf. brevicompactum elicited an unprecedented epidiketopiperazine (496), which has a trisulfide bond between the $\alpha-\beta$ positions of two amino acid residues, by adding DMSO to its natural seawater medium (Yamazaki et al., 2015b).

\section{Biosynthetic Precursor}

Biosynthetic precursor refers to one chemical that is apt to be directly incorporated into the final product. Adding various biosynthetic precursors in the fermentation medium may change biosynthesis pathways of secondary metabolites and result in the production of novel compounds (Ramm et al., 2017).

An endophytic strain Penicillium crustosum from the ripe berry of Coffea arabica L., treated with ferulic acid and quinic acid or cinnamic acid and 3,4-(methylenedioxy) cinnamic acid, was shown to produce mycophenolic acid (497) and 5-hydroxy7-methoxy-4-methylphthalide (498) (Valente et al., 2013). Three novel cytochalasins $\mathrm{Z}_{21}-\mathrm{Z}_{23} \quad(\mathbf{4 9 9 - 5 0 1 )}$ were characterized from one marine-derived fungus Chaetomium indicum KLA03 when cultivated in medium supplied by L- and D-tryptophan. Compound 498 exhibited potent cytotoxic effect on A549 cell lines with an $\mathrm{IC}_{50}$ value of $8.2 \mu \mathrm{M}$ (Wang F. Z. et al., 2011). Strain S. griseoviridis Tü 3634 could afford a wide variety of acyl $\alpha$-L-rhamnopyranosides (pyrrolyl, indolyl, thienyl, furanyl, and pyridyl derivatives) if its culture medium, respectively, added corresponding precursors, heteroaromatic carboxylic acid, benzoic acid, cinnamic acid, aminobenzoic acid, and salicylic acid (Grond et al., 2000, 2002).

\section{CONCLUSION}

Microorganisms are susceptible to culture conditions, such as medium composition, temperature, $\mathrm{pH}$, salinity, culture status, axenic or mixed culture, epigenetic modifier, biosynthetic precursor, and so on. Variation of these factors may result

\section{REFERENCES}

Adpressa, D. A., and Loesgen, S. (2016). Bioprospecting chemical diversity and bioactivity in a marine derived Aspergillus terreus. Chem. Biodivers. 13, 253-259. doi: 10.1002/cbdv.201500310

Ancheeva, E., Küppers, L., Akone, S. H., Ebrahim, W., Liu, Z., Mándi, A., et al. (2017). Expanding the metabolic profile of the fungus Chaetomium sp. through co-culture with autoclaved Pseudomonas aeruginosa. Eur. J. Org. Chem. 2017, 3256-3264. doi: 10.1002/ejoc.201700288

Araujo, F. D., Croteau, S., Slack, A. D., Milutinovic, S., Bigey, P., and Price, G. B. (2001). The DNMT1 target recognition domain resides in the $\mathrm{N}$ terminus. J. Biol. Chem. 276, 6930-6936. doi: 10.1074/jbc.M009037200

Asai, T., Chung, Y. M., Sakurai, H., Ozeki, T., Chang, F. R., Wu, Y. C., et al. (2012a). Highly oxidized ergosterols and isariotin analogs from an entomopathogenic fungus, Gibellula formosana, cultivated in the presence of epigenetic modifying agents. Tetrahedron 68, 5817-5823. doi: 10.1016/j.tet.2012. 05.020

Asai, T., Chung, Y. M., Sakurai, H., Ozeki, T., Chang, F. R., Yamashita, K., et al. (2012b). Tenuipyrone, a novel skeletal polyketide from the entomopathogenic fungus, Isaria tenuipes, cultivated in the presence of epigenetic modifiers. Org. Lett. 14, 513-515. doi: 10.1021/ol203097b in changing chemical diversity of secondary metabolites. Traditional culture method of microbe is limited to the expression of a large number of metabolic pathways that many MSMs could not be biologically synthesized. A growing body of evidence has suggested that OSMAC strategy can provide a simple, quick and effective approach for enhancing chemodiversity of MSM to obtain new drug leads through activating silent gene clusters. Moreover, employment of this strategy could avoids the waste of time and resources caused by multiple inoculation, screening, culturing and separation in comparison with mutation strategy (Fang et al., 2014) and ribosome engineering (Ochi et al., 2004). Nowadays, the rate of discovery of new MSM is getting lower and the possibility of the rediscovery of known compounds is higher than before. Therefore, OSMAC strategy would be an important alternative way to alleviate this challenge. There is a great need for new method to assist in isolating and identifying novel bioactive MSMs, such as bioassay-guided isolation, microbe genomes mining (Hug et al., 2018) and LC-MS/MS based molecular networking analysis (Wang M. et al., 2016).

\section{AUTHOR CONTRIBUTIONS}

RP made a draft of the review. XB and JC searched and collected the references. $\mathrm{HZ}$ and $\mathrm{HW}$ conceived and revised this review.

\section{FUNDING}

Financial supports from the National Key Research and Development Program of China (2018YFC0311004 and 2017YFE0103100), the National Natural Science Foundation of China (41776139 and 81773628), the Zhejiang Provincial Natural Science Foundation of China (LY16H300007 and LY16H300008), and the Hangzhou Science and Technology Development Program of China (20170432B02) were greatly appreciated.

Asai, T., Luo, D., Obara, Y., Taniguchi, T., Monde, K., Yamashita, K., et al. (2012c). Dihydrobenzofurans as cannabinoid receptor ligands from Cordyceps annullata, an entomopathogenic fungus cultivated in the presence of an HDAC inhibitor. Tetrahedron Lett. 53, 2239-2243. doi: 10.1016/j.tetlet.2012.02.088

Asai, T., Morita, S., Shirata, N., Taniguchi, T., Monde, K., Sakurai, H., et al. (2012d). Structural diversity of new C-13-polyketides produced by Chaetomium mollipilium cultivated in the presence of a $\mathrm{NAD}(+)$-dependent histone deacetylase inhibitor. Org. Lett. 14, 5456-5459. doi: 10.1021/ol302539s

Asai, T., Yamamoto, T., Chung, Y. M., Chang, F. R., Wu, Y. C., Yamashita, K., et al. (2012e). Aromatic polyketide glycosides from an entomopathogenic fungus, Cordyceps indigotica. Tetrahedron Lett. 53, 277-280. doi: 10.1016/j.tetlet.2011. 10.013

Asai, T., Yamamoto, T., and Oshima, Y. (2012f). Aromatic polyketide production in Cordyceps indigotica, an entomopathogenic fungus, induced by exposure to a histone deacetylase inhibitor. Org. Lett. 14, 2006-2009. doi: 10.1021/ol3005062

Asai, T., Morita, S., Taniguchi, T., Monde, K., and Oshima, Y. (2016). Epigenetic stimulation of polyketide production in Chaetomium cancroideum by an NAD(+)-dependent HDAC inhibitor. Org. Biomol. Chem. 14, 646-651. doi: $10.1039 / \mathrm{c} 5$ ob01595b

Asai, T., Otsuki, S., Sakurai, H., Yamashita, K., Ozeki, T., and Oshima, Y. (2013a). Benzophenones from an endophytic fungus, Graphiopsis chlorocephala, from 
Paeonia lactiflora cultivated in the presence of an NAD(+)-dependent HDAC inhibitor. Org. Lett. 15, 2058-2061. doi: 10.1021/ol400781b

Asai, T., Taniguchi, T., Yamamoto, T., Monde, K., and Oshima, Y. (2013b). Structures of spiroindicumides A and B, unprecedented carbon skeletal spirolactones, and determination of the absolute configuration by vibrational circular dichroism exciton approach. Org. Lett. 15, 4320-4323. doi: 10.1021/ ol $401741 z$

Asai, T., Yamamoto, T., Shirata, N., Taniguchi, T., Monde, K., Fujii, I., et al. (2013c). Structurally diverse chaetophenol productions induced by chemically mediated epigenetic manipulation of fungal gene expression. Org. Lett. 15, 3346-3349. doi: $10.1021 / \mathrm{ol} 401386 \mathrm{w}$

Asai, T., Yamamoto, T., and Oshima, Y. (2011). Histone deacetylase inhibitor induced the production of three novel prenylated tryptophan analogs in the entomopathogenic fungus, Torrubiella luteorostrata. Tetrahedron Lett. 52, 7042-7045. doi: 10.1016/j.tetlet.2011.10.020

Barros, B. A., de Oliveira, M. C. F., Mafezoli, J., Barbosa, F. G., and Rodrigues, E. (2012). Secondary metabolite production by the basidiomycete, Lentinus strigellus, under different culture conditions. Nat. Prod. Commun. 7, 771-773.

Beau, J., Mahid, N., Burda, W. N., Harrington, L., Shaw, L. N., Mutka, T., et al. (2012). Epigenetic tailoring for the production of anti-infective cytosporones from the marine fungus Leucostoma persoonii. Mar. Drugs 10, 762-774. doi: $10.3390 / \mathrm{md} 10040762$

Blunt, J. W., Copp, B. R., Keyzers, R. A., Munro, M. H. G., and Prinsep, M. R. (2015). Marine natural products. Nat. Prod. Rep. 32, 116-211. doi: 10.1039/ c4np00144c

Bode, H. B., Bethe, B., Hofs, R., and Zeeck, A. (2002). Big effects from small changes: possible ways to explore nature's chemical diversity. Chembiochemistry 3, 619-627. doi: 10.1002/1439-7633(20020703)3:7<619::AID-CBIC619>3.0. $\mathrm{CO} ; 2-9$

Bode, H. B., and Zeeck, A. (2000). Sphaerolone and dihydrosphaerolone, two bisnaphthyl-pigments from the fungus Sphaeropsidales sp. F-24' 707. Phytochemisty 54, 597-601. doi: 10.1016/s0031-9422(00)00145-x

Bohni, N., Cordero-Maldonado, M. L., Maes, J., Siverio-Mota, D., Marcourt, L., Munck, S., et al. (2013). Integration of microfractionation, qNMR and zebrafish screening for the in vivo bioassay-guided isolation and quantitative bioactivity analysis of natural products. PLoS One 8:e64006. doi: 10.1371/journal.pone. 0064006

Brzonkalik, K., Hümmer, D., Syldatk, C., and Neumann, A. (2012). Influence of pH and carbon to nitrogen ratio on mycotoxin production by Alternaria alternata in submerged cultivation. AMB Express 2:28. doi: 10.1186/2191-0855-2-28

Che, Q., Li, J., Li, D., Gu, Q., and Zhu, T. (2016). Structure and absolute configuration of drimentine I, an alkaloid from Streptomyces sp. CHQ-64. J. Antibiot. 69, 467-469. doi: 10.1038/ja.2015.133

Che, Q., Li, T., Liu, X., Yao, T., Li, J., Gu, Q., et al. (2015). Genome scanning inspired isolation of reedsmycins A-F, polyene-polyol macrolides from Streptomyces sp. CHQ-64. RSC Adv. 5, 22777-22782. doi: 10.1039/c4ra15415k

Che, Q., Zhu, T., Keyzers, R. A., Liu, X., Li, J., Gu, Q., et al. (2013). Polycyclic hybrid isoprenoids from a reed rhizosphere soil derived Streptomyces sp. CHQ-64. J. Nat. Prod. 76, 759-763. doi: 10.1021/np3008864

Che, Q., Zhu, T., Qi, X., Mándi, A., Kurtán, T., Mo, X., et al. (2012). Hybrid Isoprenoids from a reeds rhizosphere soil derived actinomycete Streptomyces sp. CHQ-64. Org. Lett. 14, 3438-3441. doi: 10.1021/ol301396h

Chen, H., Aktas, N., Konuklugil, B., Mándi, A., Daletos, G., Lin, W., et al. (2015a). A new fusarielin analogue from Penicillium sp. isolated from the mediterranean sponge Ircinia oros. Tetrahedron Lett. 56, 5317-5320. doi: 10.1016/j.tetlet.2015. 07.072

Chen, H., Daletos, G., Abdel-Aziz, M. S., Thomy, D., Dai, H., Brötz-Oesterhelt, H., et al. (2015b). Inducing secondary metabolite production by the soil-dwelling fungus Aspergillus terreus through bacterial co-culture. Phytochem. Lett. 12, 35-41. doi: 10.1016/j.phytol.2015.02.009

Chen, H. J., Awakawa, T., Sun, J. Y., Wakimoto, T., and Abe, I. (2013). Epigenetic modifier-induced biosynthesis of novel fusaric acid derivatives in endophytic fungi from Datura stramonium L. Nat. Prod. Bioprospect. 3, 20-23. doi: 10.1007/ s13659-013-0010-2

Chen, M., Zhang, W., Shao, C. L., Chi, Z. M., and Wang, C. Y. (2016). DNA methyltransferase inhibitor induced fungal biosynthetic products: diethylene glycol phthalate ester oligomers from the marine-derived fungus Cochliobolus lunatus. Mar. Biotechnol. 18, 409-417. doi: 10.1007/s10126-016-9703-y
Christian, O. E., Compton, J., Christian, K. R., Mooberry, S. L., Valeriote, F. A., and Crews, P. (2005). Using jasplakinolide to turn on pathways that enable the isolation of new chaetoglobosins from Phomopsis asparagi. J. Nat. Prod. 68, 1592-1597. doi: 10.1021/np050293f

Chung, Y. M., Elshazly, M., Chuang, D. W., Hwang, T. L., Asai, T., Oshima, Y., et al. (2013a). Suberoylanilide hydroxamic acid, a histone deacetylase inhibitor, induces the production of anti-inflammatory cyclodepsipeptides from Beauveria felina. J. Nat. Prod. 76, 1260-1266. doi: 10.1021/np400143j

Chung, Y. M., Wei, C. K., Chuang, D. W., El-Shazly, M., Hsieh, C. T., Asai, T., et al. (2013b). An epigenetic modifier enhances the production of anti-diabetic and anti-inflammatory sesquiterpenoids from Aspergillus sydowii. Bioorg. Med. Chem. 21, 3866-3872. doi: 10.1016/j.bmc.2013.04.004

Cole, P. A. (2008). Chemical probes for histone-modifying enzymes. Nat. Chem. Biol. 4, 590-597. doi: 10.1038/nchembio.111

Cui, C. B., Kakeya, H., and Osada, H. (1996). Novel mammalian cell cycle inhibitors, spirotryprostatins A and B, produced by Aspergillus fumigatus, which inhibit mammalian cell cycle at G2/M phase. Tetrahedron 52, 12651-12666. doi: 10.1016/0040-4020(96)00737-5

Dashti, Y., Grkovic, T., Abdelmohsen, U. R., Hentschel, U., and Quinn, R. J. (2014). Production of induced secondary metabolites by a co-culture of spongeassociated actinomycetes, Actinokineospora sp. EG49 and Nocardiopsis sp. RV163. Mar. Drugs 12, 3046-3059. doi: 10.3390/md12053046

Degenkolb, T., Heinze, S., Schlegel, B., Strobel, G., and Grafe, U. (2002). Formation of new lipoaminopeptides, acremostatins A, B, and C, by co-cultivation of Acremonium sp. tbp-5 and Mycogone rosea DSM 12973. Biosci. Biotechnol. Biochem. 66, 883-886. doi: 10.1271/bbb.66.883

Deng, Z. L., Du, C. X., Li, X., Hu, B., Kuang, Z. K., Wang, R., et al. (2013). Exploring the biologically relevant chemical space for drug discovery. J. Chem. Inf. Model. 53, 2820-2828. doi: 10.1021/ci400432a

Dinarvand, M., Rezaee, M., Masomian, M., Jazayeri, S. D., Zareian, M., Abbasi, S., et al. (2013). Effect of $\mathrm{C} / \mathrm{N}$ ratio and media optimization through response surface methodology on simultaneous productions of intra- and extracellular inulinase and invertase from Aspergillus niger ATCC 20611. Biomed Res. Int. 2013:508968. doi: 10.1155/2013/508968

Ding, G., Liu, S. C., Guo, L. D., Zhou, Y. G., and Che, Y. S. (2008). Antifungal metabolites from the plant endophytic fungus Pestalotiopsis foedan. J. Nat. Prod. 71, 615-618. doi: 10.1021/np070590f

Ding, G., Qi, Y., Liu, S., Guo, L., and Chen, X. (2012). Photipyrones A and B, new pyrone derivatives from the plant endophytic fungus Pestalotiopsis photiniae. J. Antibiot. 65, 271-273. doi: 10.1038/ja.2012.14

Ding, G., Zheng, Z. H., Liu, S. C., Zhang, H., Guo, L. D., and Che, Y. S. (2009). Photinides A-F, cytotoxic benzofuranone-derived gamma-lactones from the plant endophytic fungus Pestalotiopsis photiniae. J. Nat. Prod. 72, 942-945. doi: $10.1021 / \mathrm{np} 900084 \mathrm{~d}$

Ding, H., Wang, J. N., Zhang, D. S., and Ma, Z. J. (2017). Derivatives of holomycin and cyclopropaneacetic acid from Streptomyces sp. DT-A37. Chem. Biodivers. 14:e1700140. doi: $10.1002 / \mathrm{cbdv} .201700140$

Dong, Y. S., Lin, J., Lu, X. H., Zheng, Z. H., Ren, X., Zhang, H., et al. (2009). Cathepsin B inhibitory tetraene lactones from the fungus Talaromyces wortmannii. Helv. Chim. Acta 92, 567-574. doi: 10.1002/hlca.200800333

Dong, Y. S., Yang, J. S., Zhang, H., Lin, J., Ren, X., Liu, M., et al. (2006). Wortmannilactones A-D, 22-membered triene macrolides from Talaromyces wortmannii. J. Nat. Prod. 69, 128-130. doi: 10.1021/np0502894

Du, L., Feng, T., Zhao, B. Y., Li, D. H., Cai, S. X., Zhu, T. J., et al. (2010). Alkaloids from a deep ocean sediment-derived fungus Penicillium sp and their antitumor activities. J. Antibiot. 63, 165-170. doi: 10.1038/ja.2010.11

Du, L., King, J. B., and Cichewicz, R. H. (2014). Chlorinated polyketide obtained from a Daldinia sp. treated with the epigenetic modifier suberoylanilide hydroxamic acid. J. Nat. Prod. 77, 2454-2458. doi: 10.1021/np500522z

Du, L., Li, D. H., Zhu, T. J., Cai, S. X., Wang, F. P., Xiao, X., et al. (2009). New alkaloids and diterpenes from a deep ocean sediment derived fungus Penicillium sp. Tetrahedron 65, 1033-1039. doi: 10.1016/j.tet.2008.11.078

Ebrahim, W., El-Neketi, M., Lewald, L. I., Orfali, R. S., Lin, W. H., Rehberg, N., et al. (2016). Metabolites from the fungal endophyte Aspergillus austroafricanus in axenic culture and in fungal-bacterial mixed cultures. J. Nat. Prod. 79, 914-922. doi: 10.1021/acs.jnatprod.5b00975

Elnaggar, M. S., Ebada, S. S., Ashour, M. L., Ebrahim, W., Müller, W. E. G., Mándi, A., et al. (2016). Xanthones and sesquiterpene derivatives from a 
marine-derived fungus Scopulariopsis sp. Tetrahedron 72, 2411-2419. doi: 10. 1016/j.tet.2016.03.073

Elnaggar, M. S., Ebada, S. S., Ashour, M. L., Ebrahim, W., Singab, A., Lin, W., et al. (2017). Two new triterpenoids and a new naphthoquinone derivative isolated from a hard coral-derived fungus Scopulariopsis sp. Fitoterapia 116, 126-130. doi: 10.1016/j.fitote.2016.12.003

Elsayed, S. S., Trusch, F., Deng, H., Raab, A., Prokes, I., Busarakam, K., et al. (2015). Chaxapeptin, a lasso peptide from extremotolerant Streptomyces leeuwenhoekii strain C58 from the hyperarid Atacama desert. J. Org. Chem. 80, 10252-10260. doi: $10.1021 /$ acs.joc.5b01878

Ezaki, M., Iwami, M., Yamashita, M., Komori, T., Umehara, K., and Imanaka, H. (1992). Biphenomycin A production by a mixed culture. Appl. Environ. Microbiol. 58, 3879-3882.

Ezaki, M., Shigematsu, N., Yamashita, M., Komori, T., Umehara, K., and Imanaka, H. (1993). Biphenomycin C, a precursor of biphenomycin A in mixed culture. J. Antibiot. 46, 135-140. doi: 10.7164/antibiotics.46.135

Fang, S. M., Wu, C. J., Li, C. W., and Cui, C. B. (2014). A practical strategy to discover new antitumor compounds by activating silent metabolite production in fungi by diethyl sulphate mutagenesis. Mar. Drugs 12, 1788-1814. doi: 10. 3390/md12041788

Feller, G., Narinx, E., Arpigny, J. L., Zekhnini, Z., Swings, J., and Gerday, C. (1994). Temperature dependence of growth, enzyme secretion and activity of psychrophilic antarctic bacteria. Appl. Microbiol. Biotechnol. 41, 477-479. doi: 10.1007/BF00939039

Fuchser, J., Thiericke, R., and Zeeck, A. (1995). Biosynthesis of aspinonene, a branched pentaketide produced by Aspergillus ochraceus, Related to aspyrone. J. Chem. Soc. Perkin Trans. 1, 1663-1666. doi: 10.1039/p19950001663

Fukuda, H., Sano, N., Muto, S., and Horikoshi, M. (2006). Simple histone acetylation plays a complex role in the regulation of gene expression. Brief. Funct. Genomic. Proteomic. 5, 190-208. doi: 10.1093/bfgp/ell032

Gao, H., Zhou, L., Cai, S., Zhang, G., Zhu, T., Gu, Q., et al. (2013). Diorcinols B-E, new prenylated diphenyl ethers from the marine-derived fungus Aspergillus versicolor ZLN-60. J. Antibiot. 66, 539-542. doi: 10.1038/ja. 2013.40

Gao, S. S., Li, X. M., Zhang, Y., Li, C. S., Cui, C. M., and Wang, B. G. (2011). Comazaphilones A-F, azaphilone derivatives from the marine sediment-derived fungus Penicillium commune QSD-17. J. Nat. Prod. 74, 256-261. doi: 10.1021/ np100788h

Gao, S. S., Shang, Z., Li, X. M., Li, C. S., Cui, C. M., and Wang, B. G. (2012). Secondary metabolites produced by solid fermentation of the marine-derived fungus Penicillium commune QSD-17. Biosci. Biotechnol. Biochem. 76, 358-360. doi: $10.1271 /$ bbb. 110332

Gibson, A. M., Bratchell, N., and Roberts, T. A. (1988). Predicting microbial growth: growth responses of salmonellae in a laboratory medium as affected by $\mathrm{pH}$, sodium chloride and storage temperature. Int. J. Food Microbiol. 6, 155-178. doi: 10.1016/0168-1605(88)90051-7

Grond, S., Papastavrou, I., and Zeeck, A. (2000). Studies of precursor-directed biosynthesis with streptomyces, 3 - structural diversity of 1-o-acyl alpha1-rhamnopyranosides by precursor-directed biosynthesis with Streptomyces griseoviridis. Eur. J. Org. Chem. 10, 1875-1881. doi: 10.1002/(SICI)10990690(200005)2000:10<1875::AID-EJOC1875>3.0.CO;2-G

Grond, S., Papastavrou, I., and Zeeck, A. (2002). Studies of precursor-directed biosynthesis with streptomyces, part 4. Novel alpha-L-rhamnopyranosides from a single strain of Streptomyces by supplement-induced biosynthetic steps. Eur. J. Org. Chem. 19, 3237-3242. doi: 10.1002/1099-0690(200210)2002: 19<3237::AID-EJOC3237>3.0.CO;2-T

Gulder, T. A. M., Hong, H., Correa, J., Egereva, E., Wiese, J., Imhoff, J. F., et al. (2012). Isolation, structure elucidation and total synthesis of lajollamide a from the marine fungus Asteromyces cruciatus. Mar. Drugs 10, 2912-2935. doi: 10. $3390 / \mathrm{md} 10122912$

Gunatilaka, A. A. L. (2006). Natural products from plant-associated microorganisms: distribution, structural diversity, bioactivity, and implications of their occurrence. J. Nat. Prod. 69, 509-526. doi: 10.1021/ np058128n

Guo, W., Peng, J., Zhu, T., Gu, Q., Keyzers, R. A., and Li, D. (2013). Sorbicillamines A-E, nitrogen-containing sorbicillinoids from the deep-sea-derived fungus Penicillium sp. F23-2. J. Nat. Prod. 76, 2106-2112. doi: 10.1021/np4006647
Hemphill, C. F. P., Sureechatchaiyan, P., Kassack, M. U., Orfali, R. S., Lin, W., Daletos, G., et al. (2017). OSMAC approach leads to new fusarielin metabolites from Fusarium tricinctum. J. Antibiot. 70, 726-732. doi: 10.1038/ja.2017.21

Henrikson, J. C., Hoover, A. R., Joyner, P. M., and Cichewicz, R. H. (2009). A chemical epigenetics approach for engineering the in situ biosynthesis of a cryptic natural product from Aspergillus niger. Org. Biomol. Chem. 7, 435-438. doi: 10.1039/b819208a

Hewage, R. T., Aree, T., Mahidol, C., Ruchirawat, S., and Kittakoop, P. (2014). One strain-many compounds (OSMAC) method for production of polyketides, azaphilones, and an isochromanone using the endophytic fungus Dothideomycete sp. Phytochemstry 108, 87-94. doi: 10.1016/j.phytochem.2014. 09.013

Hoshino, S., Okada, M., Awakawa, T., Asamizu, S., Onaka, H., and Abe, I. (2017). Mycolic acid containing bacterium stimulates tandem cyclization of polyene macrolactam in a lake sediment derived rare Actinomycete. Org. Lett. 19, 4992-4995. doi: 10.1021/acs.orglett.7b02508

Hoshino, S., Okada, M., Wakimoto, T., Zhang, H., Hayashi, F., Onaka, H., et al. (2015). Niizalactams A-C, multicyclic macrolactams isolated from combined culture of Streptomyces with mycolic acid-containing bacterium. J. Nat. Prod. 78, 3011-3017. doi: 10.1021/acs.jnatprod.5b00804

Hozzein, W. N., Li, W. J., Ali, M. I., Hammouda, O., Mousa, A. S., Xu, L. H., et al. (2004). Nocardiopsis alkaliphila sp. nov., a novel alkaliphilic actinomycete isolated from desert soil in Egypt. Int. J. Syst. Evol. Microbiol. 54, 247-252. doi: 10.1099/ijs.0.02832-0

Hug, J. J., Bader, C. D., Remškar, M., Cirnski, K., and Müller, R. (2018). Concepts and methods to access novel antibiotics from actinomycetes. Antibiotics 7:E44. doi: 10.3390/antibiotics7020044

Hussain, A., Rather, M. A., Dar, M. S., Aga, M. A., Ahmad, N., Manzoor, A., et al. (2017). Novel bioactive molecules from Lentzea violacea strain AS 08 using one strain-many compounds (OSMAC) approach. Bioorg. Med. Chem. Lett. 27, 2579-2582. doi: 10.1016/j.bmcl.2017.03.075

Jiang, W., Zhong, Y. Q., Shen, L., Wu, X. D., Ye, Y., Chen, C. T. A., et al. (2014). Stress-driven discovery of natural products from extreme marine environmentKueishantao hydrothermal vent, a case study of metal switch valve. Curr. Org. Chem. 18, 925-934. doi: 10.2174/138527281807140515155705

Kakeya, H., Morishita, M., Onozawa, C., Usami, R., Horikoshi, K., Kimura, K., et al. (1997). RKS-1778, a new mammalian cell-cycle inhibitor and a key intermediate of the 11 cytochalasin group. J. Nat. Prod. 60, 669-672. doi: 10.1021/np970151o

Kamauchi, H., Kinoshita, K., Sugita, T., and Koyama, K. (2016). Conditional changes enhanced production of bioactive metabolites of marine derived fungus Eurotium rubrum. Bioorg. Med. Chem. Lett. 26, 4911-4914. doi: 10.1016/j.bmcl. 2016.09.017

Karakoç, S. B., and Aksöz, N. (2004). Optimization of carbon-nitrogen ratio for production of gibberellic acid by Pseudomonas sp. Pol. J. Microbiol. 53, 117-120.

Kozone, I., Ueda, J., Takagi, M., and Shin-ya, K. (2009). JBIR-52, a new antimycinlike compound from Streptomyces sp. ML55. J. Antibiot. 62, 593-595. doi: 10.1038/ja.2009.79

Kurosawa, K., Ghiviriga, I., Sambandan, T. G., Lessard, P. A., Barbara, J. E., Rha, C., et al. (2008). Rhodostreptomycins, antibiotics biosynthesized following horizontal gene transfer from Streptomyces padanus to Rhodococcus fascians. J. Am. Chem. Soc. 130, 1126-1127. doi: 10.1021/ja077821p

Li, C. S., An, C. Y., Li, X. M., Gao, S. S., Cui, C. M., Sun, H. F., et al. (2011). Triazole and dihydroimidazole alkaloids from the marine sediment-derived fungus Penicillium paneum SD-44. J. Nat. Prod. 74, 1331-1334. doi: 10.1021/ np200037z

Li, C. S., Li, X. M., Gao, S. S., Lu, Y. H., and Wang, B. G. (2013). Cytotoxic anthranilic acid derivatives from deep sea sediment-derived fungus Penicillium paneum SD-44. Mar. Drugs 11, 3068-3076. doi: 10.3390/md11083068

Li, X., Zvanych, R., Torchia, J., and Magarvey, N. A. (2013). Structures and biosynthesis of 12-membered macrocyclic depsipeptides from Streptomyces sp. ML55. Bioorg. Med. Chem. Lett. 23, 4150-4153. doi: 10.1016/j.bmcl.2013.05.042

Li, G., Kusari, S., Golz, C., Laatsch, H., Strohmann, C., and Spiteller, M. (2017). Epigenetic modulation of endophytic Eupenicillium sp. LG41 by a histone deacetylase inhibitor for production of decalin-containing compounds. J. Nat. Prod. 80, 983-988. doi: 10.1021/acs.jnatprod.6b00997

Li, X., Xia, Z., Tang, J., Wu, J., Tong, J., Li, M., et al. (2017). Identification and biological evaluation of secondary metabolites from marine derived fungi 
Aspergillus sp. SCSIOW3, cultivated in the presence of epigenetic modifying agents. Molecules 22:E1302. doi: 10.3390/molecules22081302

Li, Y. L., Wang, J. F., He, W. J., Lin, X. P., Zhou, X. J., and Liu, Y. H. (2017). One strain-many compounds method for production of polyketide metabolites using the sponge-derived fungus Arthrinium arundinis ZSDS1-F3. Chem. Nat. Compd. 53, 373-374. doi: 10.1007/s10600-017-1994-3

Li, J., Lu, C., and Shen, Y. (2008). Novel polyketides isolated from Streptomyces sp. Helv. Chim. Acta 91, 741-745. doi: 10.1002/hlca.200890075

Li, J., Lu, C., and Shen, Y. (2010). Macrolides of the bafilomycin family produced by Streptomyces sp. CS. J. Antibiot. 63, 595-599. doi: 10.1038/ja.2010.95

Lin, Z. J., Zhu, T. J., Chen, L., and Gu, Q. Q. (2010). Three new aspochalasin derivatives from the marine-derived fungus Spicaria elegans. Chin. Chem. Lett. 21, 824-826. doi: 10.1016/j.cclet.2010.02.019

Lin, Z. J., Zhu, T. J., Wei, H. J., Zhang, G. J., Wang, H., and Gu, Q. Q. (2009a). Spicochalasin A and new aspochalasins from the marine-derived fungus Spicaria elegans. Eur. J. Org. Chem. 18, 3045-3051. doi: 10.1002/ejoc.200801085

Lin, Z. J., Zhu, T. J., Zhang, G. J., Wei, H. J., and Gu, Q. Q. (2009b). Deoxycytochalasins from a marine-derived fungus Spicaria elegans. Can. J. Chem. 87, 486-489. doi: 10.1139/v09-006

Liu, C. X., Liu, S. H., Zhao, J. W., Zhang, J., Wang, X. J., Li, J. S., et al. (2016). A new spectinabilin derivative with cytotoxic activity from ant-derived Streptomyces sp. 1H-GS5. J. Asian Nat. Prod. Res. 19, 924-929. doi: 10.1080/10286020.2016. 1254200

Liu, M., Liu, N., Shang, F., and Huang, Y. (2016). Activation and identification of NC-1: a cryptic cyclodepsipeptide from red soil-derived Streptomyces sp. FXJ1.172. Eur. J. Org. Chem. 2016, 3943-3948. doi: 10.1002/ejoc.201600297

Liu, W. C., Yang, F., Zhang, R., Shi, X., Lu, X. H., Luan, Y. S., et al. (2016). Production of polyketides with anthelmintic activity by the fungus Talaromyces wortmannii using one strain-many compounds (OSMAC) method. Phytochem. Lett. 18, 157-161. doi: 10.1016/j.phytol.2016.10.006

Liu, L., Bruhn, T., Guo, L., Gotz, D. C., Brun, R., Stich, A., et al. (2011). Chloropupukeanolides C-E: cytotoxic pupukeanane chlorides with a spiroketal skeleton from Pestalotiopsis fici. Chemistry 17, 2604-2613. doi: 10.1002/chem. 201003129

Liu, L., Li, Y., Liu, S. C., Zheng, Z. H., Chen, X. L., Zhang, H., et al. (2009a). Chloropestolide A, an antitumor metabolite with an unprecedented spiroketal skeleton from Pestalotiopsis fici. Org. Lett. 11, 2836-2839. doi: 10.1021/ ol $901039 \mathrm{~m}$

Liu, L., Liu, S. C., Niu, S. B., Guo, L. D., Chen, X. L., and Che, Y. S. (2009b). Isoprenylated chromone derivatives from the plant endophytic fungus Pestalotiopsis fici. J. Nat. Prod. 72, 1482-1486. doi: 10.1021/np900308s

Liu, L., Liu, S. C., Jiang, L. H., Chen, X. L., Guo, L. D., and Che, Y. S. (2008a). Chloropupukeananin, the first chlorinated pupukeanane derivative, and its precursors from Pestalotiopsis fici. Org. Lett. 10, 1397-1400. doi: 10.1021/ ol800136t

Liu, L., Tian, R., Liu, S., Chen, X., Guo, L., and Che, Y. (2008b). Pestaloficiols A-E, bioactive cyclopropane derivatives from the plant endophytic fungus Pestalotiopsis fici. Bioorg. Med. Chem. 16, 6021-6026. doi: 10.1016/j.bmc.2008. 04.052

Liu, R., Lin, Z. J., Zhu, T. J., Fang, Y. C., Gu, Q. Q., and Zhu, W. M. (2008c). Novel open-chain cytochalasins from the marine-derived fungus Spicaria elegans. J. Nat. Prod. 71, 1127-1132. doi: 10.1021/np070539b

Liu, L., Niu, S., Lu, X., Chen, X., Zhang, H., Guo, L., et al. (2010). Unique metabolites of Pestalotiopsis fici suggest a biosynthetic hypothesis involving a Diels-Alder reaction and then mechanistic diversification. Chem. Commun. 46, 460-462. doi: 10.1039/b918330b

Liu, R., Gu, Q. Q., Zhu, W. M., Cui, C. B., and Fan, G. T. (2005). Trichodermamide $\mathrm{A}$ and aspergillazine A, two cytotoxic modified dipeptides from a marinederived fungus Spicaria elegans. Arch. Pharm. Res. 28, 1042-1046. doi: 10.1007/ bf02977399

Liu, R., Gu, Q. Q., Zhu, W. M., Cui, C. B., Fan, G. T., Fang, Y. C., et al. (2006). 10phenyl- 12-cytochalasins $Z(7), Z(8)$, and $Z(9)$ from the marine-derived fungus Spicaria elegans. J. Nat. Prod. 69, 871-875. doi: 10.1021/np050201m

Liu, S., Dai, H. F., Heering, C., Janiak, C., Lin, W. H., Liu, Z., et al. (2017). Inducing new secondary metabolites through co-cultivation of the fungus Pestalotiopsis sp with the bacterium Bacillus subtilis. Tetrahedron Lett. 58, 257-261. doi: 10.1016/j.tetlet.2016.12.026
Liu, S., Xu, M., Zhang, H., Qi, H., Zhang, J., Liu, C., et al. (2015). New cytotoxic spectinabilin derivative from ant-associated Streptomyces sp. 1H-GS5. J. Antibiot. 69, 128-131. doi: 10.1038/ja.2015.99

Liu, Y., Li, X. M., Meng, L. H., Jiang, W. L., Xu, G. M., Huang, C. G., et al. (2015). Bisthiodiketopiperazines and acorane sesquiterpenes produced by the marine-derived fungus Penicillium adametzioides AS-53 n different culture media. J. Nat. Prod. 78, 1294-1299. doi: 10.1021/acs.jnatprod.5b00102

Lu, C., and Shen, Y. (2003). A new macrolide antibiotic with antitumor activity produced by Streptomyces sp. CS, a commensal microbe of Maytenus hookeri. J. Antibiot. 56, 415-418. doi: 10.7164/antibiotics.56.415

Lu, C., and Shen, Y. (2004). Two new macrolides produced by Streptomyces sp. CS. J. Antibiot. 57, 597-600. doi: 10.7164/antibiotics.57.597

Luan, Y. P., Wei, H. J., Zhang, Z. P., Che, Q., Liu, Y. K., Zhu, T. J., et al. (2014). Eleganketal A, a highly oxygenated dibenzospiroketal from the marine-derived fungus Spicaria elegans KLA03. J. Nat. Prod. 77, 1718-1723. doi: 10.1021/ np500458a

Ma, L., Xing, D., Wang, H., Wang, X., and Xue, D. (2009). Effect of culture conditions on cell growth and lipid accumulation of oleaginous microorganism. Chin. J. Biotechnol. 25, 55-59.

Marmann, A., Aly, A. H., Lin, W. H., Wang, B. G., and Proksch, P. (2014). Cocultivation a powerful emerging tool for enhancing the chemical diversity of microorganisms. Mar. Drugs 12, 1043-1065. doi: 10.3390/md12021043

Meng, L. H., Li, X. M., Liu, Y., Xu, G. M., and Wang, B. G. (2017). Antimicrobial alkaloids produced by the mangrove endophyte Penicillium brocae MA231 using the OSMAC approach. RSC Adv. 7, 55026-55033. doi: 10.1039/ c7ra12081h

Meng, L. H., Li, X. M., Lv, C. T., Huang, C. G., and Wang, B. G. (2014). Brocazines A-F, cytotoxic bisthiodiketopiperazine derivatives from Penicillium brocae MA231, an endophytic fungus derived from the marine mangrove plant Avicennia marina. J. Nat. Prod. 77, 1921-1927. doi: 10.1021/np500382k

Meng, L. H., Liu, Y., Li, X. M., Xu, G. M., Ji, N. Y., and Wang, B. G. (2015a). Citrifelins A and B, citrinin adducts with a tetracyclic framework from cocultures of marine-derived isolates of Penicillium citrinum and Beauveria felina. J. Nat. Prod. 78, 2301-2305. doi: 10.1021/acs.jnatprod.5b00450

Meng, L. H., Zhang, P., Li, X. M., and Wang, B. G. (2015b). Penicibrocazines A-E, five new sulfide diketopiperazines from the marine-derived endophytic fungus Penicillium brocae. Mar. Drugs 13, 276-287. doi: 10.3390/md13010276

Miao, F. P., Liang, X. R., Liu, X. H., and Ji, N. Y. (2014). Aspewentins A-C, norditerpenes from a cryptic pathway in an algicolous strain of Aspergillus wentii. J. Nat. Prod. 77, 429-432. doi: 10.1021/ $\mathrm{np} 401047 \mathrm{w}$

Moore, J. M., Bradshaw, E., Seipke, R. F., Hutchings, M. I., and McArthur, M. (2012). Use and discovery of chemical elicitors that stimulate biosynthetic gene clusters in Streptomyces bacteria. Methods Enzymol. 517, 367-385. doi: 10.1016/ B978- 0-12-404634-4.00018-8

Ochi, K., Okamoto, S., Tozawa, Y., Inaoka, T., Hosaka, T., Xu, J., et al. (2004). Ribosome engineering and secondary metabolite production. Adv. Appl. Microbiol. 56, 155-184. doi: 10.1016/S0065-2164(04)56005-7

Oh, D. C., Jensen, P. R., Kauffman, C. A., and Fenical, W. (2005). Libertellenones A-D: induction of cytotoxic diterpenoid biosynthesis by marine microbial competition. Bioorg. Med. Chem. 13, 5267-5273. doi: 10.1016/j.bmc.2005. 05.068

Oh, D. C., Kauffman, C. A., Jensen, P. R., and Fenical, W. (2007). Induced production of emericellamides $\mathrm{A}$ and $\mathrm{B}$ from the marine-derived fungus Emericella sp. in competing co-culture. J. Nat. Prod. 70, 515-520. doi: 10.1021/ np060381f

Oikawa, H., Murakami, Y., and Ichihara, A. (1992). Useful approach to find the plausible biosynthetic precursors of secondary metabolites using P-450 inhibitors-postulated intermediates of chaetoglobosin-A1. J. Chem. Soc. Perkin Trans. 1, 2949-2953. doi: 10.1039/p19920002949

Ola, A. R., Thomy, D., Lai, D., Brotz-Oesterhelt, H., and Proksch, P. (2013). Inducing secondary metabolite production by the endophytic fungus Fusarium tricinctum through coculture with Bacillus subtilis. J. Nat. Prod. 76, 2094-2099. doi: $10.1021 / \mathrm{np} 400589 \mathrm{~h}$

Onaka, H. (2017). Novel antibiotic screening methods to awaken silent or cryptic secondary metabolic pathways in actinomycetes. J. Antibiot. 70, 865-870. doi: 10.1038/ja.2017.51 
Onaka, H., Mori, Y., Igarashi, Y., and Furumai, T. (2011). Mycolic acid-containing bacteria induce natural-product biosynthesis in Streptomyces species. Appl. Environ. Microbiol. 77, 400-406. doi: 10.1128/AEM.01337-10

Overy, D. P., Zidorn, C., Petersen, B. O., Duus, J. Ø., Dalsgaard, P. W., Larsen, T. O., et al. (2005). Medium dependant production of corymbiferone a novel product from Penicillium hordei cultured on plant tissue agar. Tetrahedron Lett. 46, 3225-3228. doi: 10.1016/j.tetlet.2005.03.043

Paranagama, P. A., Wijeratne, E. M. K., and Gunatilaka, A. A. L. (2007). Uncovering biosynthetic potential of plant-associated fungi: effect of culture conditions on metabolite production by Paraphaeosphaeria quadriseptata and Chaetomium chiversii. J. Nat. Prod. 70, 1939-1945. doi: 10.1021/np07 0504b

Park, H. B., Park, J. S., Lee, S. I., Shin, B., Oh, D. C., and Kwon, H. C. (2017). Gordonic acid, a polyketide glycoside derived from bacterial coculture of Streptomyces and Gordonia Species. J. Nat. Prod. 80, 2542-2546. doi: 10.1021/ acs.jnatprod.7b00293

Peng, J. X., Gao, H. Q., Zhang, X. M., Wang, S., Wu, C. M., Gu, Q. Q., et al. (2014). Psychrophilins E-H and versicotide C, cyclic peptides from the marinederived fungus Aspergillus versicolor ZLN-60. J. Nat. Prod. 77, 2218-2223. doi: $10.1021 / \mathrm{np} 500469 \mathrm{~b}$

Peng, X. P., Wang, Y., Liu, P. P., Hong, K., Chen, H., Yin, X., et al. (2011). Aromatic compounds from the halotolerant fungal strain of Wallemia sebi PXP-89 in a hypersaline medium. Arch. J. Pharm. Res. 34, 907-912. doi: 10.1007/s12272011-0607-0

Pérez, J., Braña, A. F., Shimkets, L. J., Sevillano, L., and Santamaría, R. I. (2011). Myxococcus xanthus induces actinorhodin overproduction and aerial mycelium formation by Streptomyces coelicolor. Microb. Biotechnol. 4, 175-183. doi: 10. 1111/j.1751-7915.2010.00208.x

Poolman, B., and Glaasker, E. (1998). Regulation of compatible solute accumulation in bacteria. Mol. Microbiol. 29, 397-407. doi: 10.1046/j.13652958.1998.00875.x

Puder, C., Loya, S., Hizi, A., and Zeeck, A. (2001). New co-metabolites of the streptazolin pathway. J. Nat. Prod. 64, 42-45. doi: 10.1021/np000377i

Ramm, S., Krawczyk, B., Mühlenweg, A., Poch, A., Mösker, E., and Süssmuth, R. D. (2017). A self-sacrificing $n$-methyltransferase is the precursor of the fungal natural product omphalotin. Angew. Chem. Int. Ed. 56, 9994-9997. doi: 10.1002/anie.201703488

Rateb, M. E., Hallyburton, I., Houssen, W. E., Bull, A. T., Goodfellow, M., Santhanam, R., et al. (2013). Induction of diverse secondary metabolites in Aspergillus fumigatus by microbial co-culture. RSC Adv. 3, 14444-14450. doi: $10.1039 /$ c3ra42378f

Rateb, M. E., Houssen, W. E., Arnold, M., Abdelrahman, M. H., Deng, H., Harrison, W. T., et al. (2011a). Chaxamycins A-D, bioactive ansamycins from a hyperarid desert Streptomyces sp. J. Nat. Prod. 74, 1491-1499. doi: 10.1021/np200 $320 \mathrm{u}$

Rateb, M. E., Houssen, W. E., Harrison, W. T. A., Deng, H., Okoro, C. K., Asenjo, J. A., et al. (2011b). Diverse metabolic profiles of a Streptomyces strain isolated from a hyper-arid environment. J. Nat. Prod. 74, 1965-1971. doi: 10.1021/ np200470u

Reid, K. A., Hamilton, J. T. G., Bowden, R. D., O’Hagan, D., Dasaradhi, L., Amin, M. R., et al. (1995). Biosynthesis of fluorinated secondary metabolites by Streptomyces cattleya. Microbiology 141, 1385-1393. doi: 10.1099/13500872141-6-1385

Ruiz, B., Chavez, A., Forero, A., Garca-Huante, Y., Romero, A., Sanchez, M., et al. (2009). Production of microbial secondary metabolites: regulation by the carbon source. Crit. Rev. Miocrbiol. 36, 146-167. doi: 10.3109/ 10408410903489576

Sato, S. (1990). Microbial production and control of cellular growth under high dissolved oxygen concentration. J. Ferment. Bioeng. 70:293. doi: 10.1016/0922$338 \mathrm{x}(90) 90076-9$

Schäberle, T. F., Orland, A., and König, G. M. (2014). Enhanced production of undecylprodigiosin in Streptomyces coelicolor by co-cultivation with the corallopyronin A-producing myxobacterium, Corallococcus coralloides. Biotechnol. Lett. 36, 641-648. doi: 10.1007/s10529-013$1406-0$

Scherlach, K., and Hertweck, C. (2009). Triggering cryptic natural product biosynthesis in microorganisms. Org. Biomol. Chem. 7, 1753-1760. doi: 10. $1039 / \mathrm{b} 821578 \mathrm{~b}$
Schneider, P., Misiek, M., and Hoffmeister, D. (2008). In vivo and in vitro production options for fungal secondary metabolites. Mol. Pharm. 5, 234-242. doi: $10.1021 / \mathrm{mp} 7001544$

Schroeckh, V., Scherlach, K., Nutzmann, H. W., Shelest, E., Schmidt-Heck, W., Schuemann, J., et al. (2009). Intimate bacterial-fungal interaction triggers biosynthesis of archetypal polyketides in Aspergillus nidulans. Proc. Natl. Acad. Sci. 106, 14558-14563. doi: 10.1073/pnas.0901870106

Senadeera, S. P., Wiyakrutta, S., Mahidol, C., Ruchirawat, S., and Kittakoop, P. (2012). A novel tricyclic polyketide and its biosynthetic precursor azaphilone derivatives from the endophytic fungus Dothideomycete sp. Org. Biomol. Chem. 10, 7220-7226. doi: 10.1039/c2ob25959a

Seyedsayamdost, M. R. (2014). High-throughput platform for the discovery of elicitors of silent bacterial gene clusters. Proc. Natl. Acad Sci. U.S.A. 111, 7266-7271. doi: 10.1073/pnas.1400019111

Shang, Z., Salim, A. A., and Capon, R. J. (2017). Chaunopyran a: cocultivation of marine mollusk-derived fungi activates a rare class of 2-alkenyl-tetrahydropyran. J. Nat. Prod. 80, 1167-1172. doi: 10.1021/acs. jnatprod.7b00144

Shin, C. S., Kim, H. J., Kim, M. J., and Ju, J. Y. (1998). Morphological change and enhanced pigment production of Monascus when cocultured with Saccharomyces cerevisiae or Aspergillus oryzae. Biotechnol. Bioeng. 59, 576-581. doi: 10.1002/(SICI)1097-0290(19980905)59:5<576::AID-BIT7>3.0.CO;2-7

Singh, V., Haque, S., Niwas, R., Srivastava, A., Pasupuleti, M., and Tripathi, C. K. M. (2017). Strategies for fermentation medium optimization: an indepth review. Front. Microbiol. 7:2087. doi: 10.3389/fmicb.2016.02087

Siridechakorn, I., Yue, Z., Mittraphab, Y., Lei, X., and Pudhom, K. (2017). Identification of spirobisnaphthalene derivatives with antitumor activities from the endophytic fungus Rhytidhysteron rufulum AS21B. Bioorg. Med. Chem. 25, 2878-2882. doi: 10.1016/j.bmc.2017. 02.054

Suh, J. H., and Shin, C. S. (2000a). Analysis of the morphologic changes of Monascus sp J101 cells cocultured with Saccharomyces cerevisiae. FEMS Microbiol. Lett. 193, 143-147. doi: 10.1016/s0378-1097(00) 00470-5

Suh, J. H., and Shin, C. S. (2000b). Physiological analysis on novel coculture of Monascus sp J101 with Saccharomyces cerevisiae. FEMS Microbiol. Lett. 190, 241-245. doi: 10.1111/j.1574-6968.2000.tb09293.x

Sun, J., Awakawa, T., Noguchi, H., and Abe, I. (2012). Induced production of mycotoxins in an endophytic fungus from the medicinal plant Datura stramonium L. Bioorg. Med. Chem. Lett. 22, 6397-6400. doi: 10.1016/j.bmcl. 2012.08.063

Sureram, S., Kesornpun, C., Mahidol, C., Ruchirawat, S., and Kittakoop, P. (2013). Directed biosynthesis through biohalogenation of secondary metabolites of the marine-derived fungus Aspergillus unguis. RSC Adv. 3, 1781-1788. doi: 10.1039/ c2ra23021f

Sureram, S., Wiyakrutta, S., Ngamrojanavanich, N., Mahidol, C., Ruchirawat, S., and Kittakoop, P. (2012). Depsidones, aromatase inhibitors and radical scavenging agents from the marine-derived fungus Aspergillus unguis CRI28203. Planta Med. 78, 582-588. doi: 10.1055/s-0031-1298228

Tammen, S. A., Friso, S., and Choi, S.-W. (2013). Epigenetics: the link between nature and nurture. Mol. Aspects Med. 34, 753-764. doi: 10.1016/j.mam.2012. 07.018

Tan, Y., Wang, Z., and Marshall, K. C. (1998). Modeling pH effects on microbial growth: a statistical thermodynamic approach. Biotechnol. Bioeng. 59, 724-731. doi: 10.1002/(SICI) 1097-0290(19980920)59:6<724::AID-BIT9>3.0.CO;2-H

Tang, J. W., Wang, W. G., Li, A., Yan, B. C., Chen, R., Li, X. N., et al. (2017). Polyketides from the endophytic fungus Phomopsis sp sh917 by using the one strain/many compounds strategy. Tetrahedron 73, 3577-3584. doi: 10.1016/j. tet.2017.02.019

Teles, A. P., and Takahashi, J. A. (2013). Paecilomide, a new acetylcholinesterase inhibitor from Paecilomyces lilacinus. Microbiol. Res. 168, 204-210. doi: 10. 1016/j.micres.2012.11.007

Thorneley, R. N. F. (1990). Metal Ions and Bacteria, Vol. 8. Amsterdam: Elsevier Ltd, 298-299. doi: 10.1016/0167-7799(90)90204-B

Traxler, M. F., Watrous, J. D., Alexandrov, T., Dorrestein, P. C., and Kolter, R. (2013). Interspecies interactions stimulate diversification of the Streptomyces coelicolor secreted metabolome. mBio 4:e00459-13. doi: 10.1128/mBio. 00459-13 
Uchida, I., Uchida, I., Shigematsu, N., Shigematsu, N., Ezaki, M., Ezaki, M., et al. (1985). Biphenomycins A and B, novel peptide antibiotics II. Structural elucidation of biphenomycins A and B. J. Antibiot. 38, 1462-1468. doi: 10.7164/ antibiotics.38.1462

Uchoa, P. K. S., Pimenta, A. T. A., Braz-Filho, R., de Oliveira, M., Saraiva, N. N., Rodrigues, B. S. F., et al. (2017). New cytotoxic furan from the marine sedimentderived fungi Aspergillus niger. Nat. Prod. Res. 31, 2599-2603. doi: 10.1080/ 14786419.2017.1283499

Ueda, J., Nagai, A., Izumikawa, M., Chijiwa, S., Takagi, M., and Shin-ya, K. (2008). A novel antimycin-like compound, JBIR-06, from Streptomyces sp. ML55. J. Antibiot. 61, 241-244. doi: 10.1038/ja.2008.35

Ueda, J., Togashi, T., Matukura, S., Nagai, A., Nakashima, T., Komaki, H., et al. (2007). A novel nuclear export inhibitor JBIR-02, a new piericidin discovered from Streptomyces sp. ML55. J. Antibiot. 60, 459-462. doi: 10.1038/ja. 2007.59

Valente, A., Ferreira, A. G., Daolio, C., Rodrigues, E., Boffo, E. F., Souza, A. Q. L., et al. (2013). Production of 5-hydroxy-7-methoxy-4-methylphthalide in a culture of Penicillium crustosum. An. Acad. Bras. Cienc. 85, 487-496. doi: 10. 1590/s0001-37652013005000024

Vervoort, H. C., Draskovic, M., and Crews, P. (2011). Histone deacetylase inhibitors as a tool to up-regulate new fungal biosynthetic products: isolation of EGM-556, a cyclodepsipeptide, from Microascus sp. Org. Lett. 13, 410-413. doi: $10.1021 /$ ol1027199

Wakefield, J., Hassan, H. M., Jaspars, M., Ebel, R., and Rateb, M. E. (2017). Dual induction of new microbial secondary metabolites by fungal bacterial co-cultivation. Front. Microbiol. 8:1284. doi: 10.3389/fmicb.2017.01284

Wang, B., Park, E. M., King, J. B., Mattes, A. O., Nimmo, S. L., Clendinen, C., et al. (2015a). Transferring fungi to a deuterium-enriched medium results in assorted, conditional changes in secondary metabolite production. J. Nat. Prod. 78, 1415-1421. doi: 10.1021/acs.jnatprod.5b00337

Wang, F. Z., Wei, H. J., Zhu, T. J., Li, D. H., Lin, Z. J., and Gu, Q. Q. (2011). Three new cytochalasins from the marine-derived fungus Spicaria elegans KLA03 by supplementing the cultures with $L$ - and $D$-tryptophan. Chem. Biodivers. 8 , 887-894. doi: 10.1002/cbdv.201000133

Wang, J., Wang, Z., Ju, Z. R., Wan, J. T., Liao, S. R., Lin, X. P., et al. (2015b). Cytotoxic cytochalasins from marine-derived fungus Arthrinium arundinis. Planta Med. 81, 160-166. doi: 10.1055/s-0034-1383403

Wang, J., Wei, X. Y., Qin, X. C., Lin, X. P., Zhou, X. F., Liao, S. R., et al. (2015c). Arthpyrones A-C, pyridone alkaloids from a sponge-derived fungus Arthrinium arundinis ZSDS1-F3. Org. Lett. 17, 656-659. doi: 10.1021/ol503646c

Wang, J. F., Xu, F. Q., Wang, Z., Lu, X., Wan, J. T., Yang, B., et al. (2014). A new naphthalene glycoside from the sponge-derived fungus Arthrinium sp ZSDS1-F3. Nat. Prod. Res. 28, 1070-1074. doi: 10.1080/14786419.2014.905935

Wang, Q. X., Bao, L., Yang, X. L., Guo, H., Ren, B., Guo, L. D., et al. (2013a). Tricycloalternarenes F-H: three new mixed terpenoids produced by an endolichenic fungus Ulocladium sp. using OSMAC method. Fitoterapia 85, 8-13. doi: 10.1016/j.fitote.2012.12.029

Wang, Q. X., Bao, L., Yang, X. L., Guo, H., Yang, R. N., Ren, B. A., et al. (2012). Polyketides with antimicrobial activity from the solid culture of an endolichenic fungus Ulocladium sp. Fitoterapia 83, 209-214. doi: 10.1016/j.fitote.2011.10.013

Wang, Q. X., Bao, L., Yang, X. L., Liu, D. L., Guo, H., Dai, H. Q., et al. (2013b). Ophiobolins P-T, five new cytotoxic and antibacterial sesterterpenes from the endolichenic fungus Ulocladium sp. Fitoterapia 90, 220-227. doi: 10.1016/j. fitote.2013.08.002

Wang, Y., Lu, Z., Sun, K., and Zhu, W. (2011). Effects of high salt stress on secondary metabolite production in the marine-derived fungus Spicaria elegans. Mar. Drugs 9, 535-542. doi: 10.3390/md9040535

Wang, Z., Fu, P., Liu, P. P., Wang, P., Hou, J. B., Li, W. J., et al. (2013c). New pyran-2-ones from alkalophilic actinomycete, Nocardiopsis alkaliphila sp. Nov. YIM-80379. Chem. Biodivers. 10, 281-287. doi: 10.1002/cbdv.201200086

Wang, L., Li, M., Tang, J., and Li, X. (2016). Eremophilane sesquiterpenes from a deep marine-derived fungus, Aspergillus sp. SCSIOW2, cultivated in the presence of epigenetic modifying agents. Molelules 21:473. doi: 10.3390/ molecules21040473

Wang, M., Carver, J. J., Phelan, V. V., Sanchez, L. M., Garg, N., Peng, Y., et al. (2016). Sharing and community curation of mass spectrometry data with global natural products social molecular networking. Nat. Biotechnol. 34, 828-837. doi: $10.1038 /$ nbt.3597
Wang, X.E., You, J. L., King, J. B., Powell, D. R., and Cichewicz, R. H. (2012). Waikialoid A suppresses hyphal morphogenesis and inhibits biofilm development in pathogenic Candida albicans. J. Nat. Prod. 75, 707-715. doi: $10.1021 / \mathrm{np} 2009994$

Wang, W. J., Li, D. Y., Li, Y. C., Hua, H. M., Ma, E. L., and Li, Z. L. (2014). Caryophyllene sesquiterpenes from the marine-derived fungus Ascotricha sp ZJ-M-5 by the one strain-many compounds strategy. J. Nat. Prod. 77, 13671371. doi: $10.1021 / \mathrm{np} 500110 \mathrm{z}$

Wang, X. R., Sena, J. G., Hoover, A. R., King, J. B., Ellis, T. K., Powell, D. R., et al. (2010). Chemical epigenetics alters the secondary metabolite composition of guttate excreted by an atlantic-forest-soil-derived Penicillium citreonigrum. J. Nat. Prod. 73, 942-948. doi: 10.1021/np100142h

Wasil, Z., Pahirulzaman, K. A. K., Butts, C., Simpson, T. J., Lazarus, C. M., and Cox, R. J. (2013). One pathway, many compounds: heterologous expression of a fungal biosynthetic pathway reveals its intrinsic potential for diversity. Chem. Sci. 4, 3845-3856. doi: 10.1039/c3sc51785c

Wijeratne, E. M. K., Carbonezi, C. A., Takahashi, J. A., Seliga, C. J., Turbyville, T. J., Pierson, E. E., et al. (2004). Isolation, optimization of production and structure-activity relationship studies of monocillin I, the cytotoxic constituent of Paraphaeosphaeria quadriseptata. J. Antibiot. 57, 541-546. doi: 10.7164/ antibiotics.57.541

Wijesekera, K., Mahidol, C., Ruchirawat, S., and Kittakoop, P. (2017). Metabolite diversification by cultivation of the endophytic fungus Dothideomycete $\mathrm{sp}$. in halogen containing media: cultivation of terrestrial fungus in seawater. Bioorg. Med. Chem. 25, 2868-2877. doi: 10.1016/j.bmc.2017.03.040

Williams, R. B., Henrikson, J. C., Hoover, A. R., Lee, A. E., and Cichewicz, R. H. (2008). Epigenetic remodeling of the fungal secondary metabolome. Org. Biomol. Chem. 6, 1895-1897. doi: 10.1039/b804701d

Wu, C., Zacchetti, B., Ram, A. F., van Wezel, G. P., Claessen, D., and Hae Choi, Y. (2015). Expanding the chemical space for natural products by Aspergillusstreptomyces co-cultivation and biotransformation. Sci. Rep. 5:10868. doi: 10. 1038/srep 10868

Wu, G., Sun, X., Yu, G., Wang, W., Zhu, T., Gu, Q., et al. (2014). Cladosins A-E, hybrid polyketides from a deep-sea-derived fungus, Cladosporium sphaerospermum. J. Nat. Prod. 77, 270-275. doi: 10.1021/np400833x

Xie, L. R., Li, D. Y., Li, Z. L., Hua, H. M., Wang, P. L., and Wu, X. (2013a). A new cyclonerol derivative from a marine-derived fungus Ascotricha sp. ZJ-M-5. Nat. Prod. Res. 27, 847-850. doi: 10.1080/14786419.2012.711327

Xie, L. R., Li, D. Y., Wang, P. L., Hua, H. M., Wu, X., and Li, Z. L. (2013b). A new 3, 4-seco-lanostane triterpenoid from a marine-derived fungus Ascotricha sp. ZJ-M-5. Acta Pharm. Sin. 48, 89-93.

Yang, X. L., Awakawa, T., Wakimoto, T., and Abe, I. (2013). Induced production of novel prenyldepside and coumarins in endophytic fungi Pestalotiopsis acaciae. Tetrahedron Lett. 54, 5814-5817. doi: 10.1016/j.tetlet.2013.08.054

Yamazaki, H., Rotinsulu, H., Narita, R., Takahashi, R., and Namikoshi, M. (2015a). Induced production of halogenated epidithiodiketopiperazines by a marinederived Trichoderma cf. brevicompactum with sodium halides. J. Nat. Prod. 78, 2319-2321. doi: 10.1021/acs.jnatprod.5b00669

Yamazaki, H., Takahashi, O., Murakami, K., and Namikoshi, M. (2015b). Induced production of a new unprecedented epitrithiodiketopiperazine, chlorotrithiobrevamide, by a culture of the marine-derived Trichoderma cf. brevicompactum with dimethyl sulfoxide. Tetrahedron Lett. 56, 6262-6265. doi: 10.1016/j.tetlet.2015.09.113

Yang, D., Liu, F., and Yang, X. (2017). DNA methyltransferase inhibitor dramatically alters the secondary metabolism of Pestalotiopsis microspora. J. Chin. Pharm. Sci. 5, 355-359. doi: 10.5246/jcps.2017. 05.037

Yang, X. L., Huang, L., and Ruan, X. L. (2014). Epigenetic modifiers alter the secondary metabolite composition of a plant endophytic fungus, Pestalotiopsis crassiuscula obtained from the leaves of Fragaria chiloensis. J. Asian Nat. Prod. Res. 16, 412-417. doi: 10.1080/10286020.2014. 881356

Yang, X. L., and Li, Z. Z. (2013). New spiral gamma-lactone enantiomers from the plant endophytic fungus Pestalotiopsis foedan. Molecules 18, 2236-2242. doi: 10.3390/molecules 18022236

Yang, Y., Fu, X., Li, L., Zeng, Y., Li, C., He, Y., et al. (2012). Naphthomycins L-N, ansamycin antibiotics from Streptomyces sp. CS. J. Nat. Prod. 75, 1409-1413. doi: $10.1021 / \mathrm{np} 300109$ s 
Yu, G. H., Wu, G. W., Zhu, T. J., Gu, Q. Q., and Li, D. H. (2015). Cladosins F and G, two new hybrid polyketides from the deep-sea-derived Cladosporium sphaerospermum 2005-01-E3. J. Asian Nat. Prod. Res. 17, 120-124. doi: 10.1080/ 10286020.2014 .940330

Yuan, C., Guo, Y. H., Wang, H. Y., Ma, X. J., Jiang, T., Zhao, J. L., et al. (2016). Allelopathic polyketides from an endolichenic fungus Myxotrichum sp. by using OSMAC strategy. Sci. Rep. 6:19350. doi: 10.1038/srep19350

Yuan, C., Wang, H. Y., Wu, C. S., Jiao, Y., Li, M., Wang, Y. Y., et al. (2013). Austdiol, fulvic acid and citromycetin derivatives from an endolichenic fungus, Myxotrichum sp. Phytochem. Lett. 6, 662-666. doi: 10.1016/j.phytol.2013.08.011

Zhang, H., Ruan, C., Bai, X., Chen, J., and Wang, H. (2018a). Heterocyclic alkaloids as antimicrobial agents of Aspergillus fumigatus D endophytic on Edgeworthia chrysantha. Chem. Nat. Compd. 54, 411-414. doi: 10.1007/s10600-018-2365-4

Zhang, H., Zhao, Z., Chen, J., Bai, X., and Wang, H. (2018b). Tricycloalternarene analogs from a symbiotic fungus Aspergillus sp. D and their antimicrobial and cytotoxic effects. Molecules 23, 855-861. doi: 10.3390/molecules23040855

Zhang, L., Niaz, S. I., Khan, D., Wang, Z., Zhu, Y., Zhou, H., et al. (2017a). Induction of diverse bioactive secondary metabolites from the mangrove endophytic fungus Trichoderma sp. (strain 307) by co-cultivation with Acinetobacter johnsonii (strain B2). Mar. Drugs 15:35. doi: 10.3390/md15020035

Zhang, Z., He, X., Zhang, G., Che, Q., Zhu, T., Gu, Q., et al. (2017b). Inducing secondary metabolite production by combined culture of Talaromyces aculeatus and Penicillium variabile. J. Nat. Prod. 80, 3167-3171. doi: 10.1021/acs.jnatprod. $7 \mathrm{~b} 00417$

Zhang, Z., Chen, L., Zhang, X., Liang, Y., Anjum, K., Chen, L., et al. (2017c). Bioactive bafilomycins and a new N-Arylpyrazinone derivative from marinederived Streptomyces sp. HZP-2216E. Planta Med. 83, 1405-1411. doi: 10.1055/ s-0043-111897

Zhang, X., Chen, L., Chai, W., Lian, X. Y., and Zhang, Z. (2017d). A unique indolizinium alkaloid streptopertusacin $\mathrm{A}$ and bioactive bafilomycins from marine-derived Streptomyces sp. HZP-2216E. Phytochemistry 144, 119-126. doi: 10.1016/j.phytochem.2017.09.010

Zhang, Q., Wang, S. Q., Tang, H. Y., Li, X. J., Zhang, L., Xiao, J., et al. (2013). Potential allelopathic indole diketopiperazines produced by the plant endophytic Aspergillus fumigatus using the one strain-many compounds method. J. Agric. Food Chem. 61, 11447-11452. doi: 10.1021/jf403200g
Zhao, Q., Wang, G. Q., Chen, G. D., Hu, D., Li, X. X., Guo, L. D., et al. (2015). Nodulisporisteroids C-L, new 4-methyl-progesteroid derivatives from Nodulisporium sp. Steroids 102, 101-109. doi: 10.1016/j.steroids.2015.08.004

Zheng, Q. C., Chen, G. D., Kong, M. Z., Li, G. Q., Cui, J. Y., Li, X. X., et al. (2013). Nodulisporisteriods A and B, the first 3,4-seco-4-methyl-progesteroids from Nodulisporium sp. Steroids 78, 896-901. doi: 10.1016/j.steroids.2013.05.007

Zheng, Y., Zhao, B., Lu, C., Lin, X., Zheng, Z., and Su, W. (2009). Isolation, structure elucidation and apoptosis-inducing activity of new compounds from the edible fungus Lentinus striguellus. Nat. Prod. Commun. 4, 501-506.

Zhou, L. N., Gao, H. Q., Cai, S. X., Zhu, T. J., Gu, Q. Q., and Li, D. H. (2011). Two new cyclic pentapeptides from the marine-derived fungus Aspergillus versicolor. Helv. Chim. Acta 94, 1065-1070. doi: 10.1002/hlca.2010 00408

Zhu, F., Chen, G. Y., Chen, X., Huang, M. Z., and Wan, X. Q. (2011). Aspergicin, a new antibacterial alkaloid produced by mixed fermentation of two marinederived mangrove epiphytic fungi. Chem. Nat. Compd. 47, 767-769. doi: 10. 1007/s10600-011-0053-8

Zhu, F., and Lin, Y. (2006). Marinamide, a novel alkaloid and its methyl ester produced by the application of mixed fermentation technique to two mangrove endophytic fungi from the south china sea. Sci. Bull. 51, 1426-1430. doi: 10. 1007/s11434-006-1426-4

Zuck, K. M., Shipley, S., and Newman, D. J. (2011). Induced production of $N$-formyl alkaloids from Aspergillus fumigatus by co-culture with Streptomyces peucetius. J. Nat. Prod. 74, 1653-1657. doi: 10.1021/np200 $255 \mathrm{f}$

Conflict of Interest Statement: The authors declare that the research was conducted in the absence of any commercial or financial relationships that could be construed as a potential conflict of interest.

Copyright (c) 2019 Pan, Bai, Chen, Zhang and Wang. This is an open-access article distributed under the terms of the Creative Commons Attribution License (CC BY). The use, distribution or reproduction in other forums is permitted, provided the original author(s) and the copyright owner(s) are credited and that the original publication in this journal is cited, in accordance with accepted academic practice. No use, distribution or reproduction is permitted which does not comply with these terms. 